\title{
GPR37 regulates macrophage phagocytosis and resolution of inflammatory pain
}

\author{
Sangsu Bang, ${ }^{1}$ Ya-Kai Xie, ${ }^{2}$ Zhi-Jun Zhang, ${ }^{2}$ Zilong Wang, ${ }^{1}$ Zhen-Zhong Xu, ${ }^{1,2}$ and Ru-Rong ji1,3 \\ 'Department of Anesthesiology, Duke University Medical Center, Durham, North Carolina, USA. ²Department of Physiology, Center of Neuroscience, Key Laboratory of Medical Neurobiology of the Ministry of \\ Health of China, Zhejiang University School of Medicine, Hangzhou, China. ${ }^{3}$ Department of Neurobiology, Duke University Medical Center, Durham, North Carolina, USA.
}

\begin{abstract}
The mechanisms of pain induction by inflammation have been extensively studied. However, the mechanisms of pain resolution are not fully understood. Here, we report that GPR37, expressed by macrophages (MФs) but not microglia, contributes to the resolution of inflammatory pain. Neuroprotectin D1 (NPD1) and prosaptide TX14 increase intracellular $\mathrm{Ca}^{2+}\left(\mathrm{iCa}^{2+}\right)$ levels in GPR37-transfected HEK293 cells. NPD1 and TX14 also bind to GPR37 and cause GPR37-dependent $\mathrm{iCa}^{2+}$ increases in peritoneal MФs. Activation of CPR37 by NPD1 and TX14 triggers MФ phagocytosis of zymosan particles via calcium signaling. Hind paw injection of $\mathrm{pH}$-sensitive zymosan particles not only induces inflammatory pain and infiltration of neutrophils and MФs, but also causes GPR37 upregulation in MФs, phagocytosis of zymosan particles and neutrophils by MФs in inflamed paws, and resolution of inflammatory pain in WT mice. Mice lacking Gpr37 display deficits in $\mathrm{M \Phi}$ phagocytic activity and delayed resolution of inflammatory pain. Gpr37-deficient MФs also show dysregulations of proinflammatory and antiinflammatory cytokines. МФ depletion delays the resolution of inflammatory pain. Adoptive transfer of WT but not Cpr37-deficient MФs promotes the resolution of inflammatory pain. Our findings reveal a previously unrecognized role of CPR37 in regulating MФ phagocytosis and inflammatory pain resolution.
\end{abstract}

\section{Introduction}

Inflammation is characterized by 5 cardinal signs: rubor (redness), calor (increased heat), tumor (swelling), dolor (pain), and functio laesa (loss of function). As a cardinal feature of inflammation, pain protects the inflamed tissue by sending a warning (nociceptive) signal to the brain and triggering a withdrawal response. Activation of nociceptive neurons (nociceptors) in the PNS is also known to cause neurogenic inflammation and neuroinflammation by producing neuropeptides (e.g., substance P) and chemokines (e.g., CCL2) $(1,2)$. However, the ablation of nociceptive neurons potentiates bacteria-induced inflammation (3). Inflammation after tissue injury causes sequential infiltration of immune cells, including neutrophils (within hours) and macrophages (MФs) (within days), into the damaged tissue (4). These immune cells produce inflammatory mediators such as proinflammatory cytokines (TNF, IL-1 $\beta$ ) that elicit inflammatory pain by binding to cytokine receptors on nociceptors throughout skin, muscle, and joint tissues $(5,6)$.

The function of inflammation is to eliminate the initial cause of cellular injury, leading to tissue repair and a return to homeostasis. The resolution of acute inflammation is an active process involving the production of specialized proresolving mediators (SPMs), such as resolvins and maresins, which are biosynthesized from omega-3 unsaturated fatty acids. SPMs potently suppress

Related Commentary: p. 3246

Authorship note: SB, YKX, and ZJZ contributed equally to this work. Conflict of interest: The authors have declared that no conflict of interest exists. Submitted: January 18, 2018; Accepted: May 9, 2018.

Reference information: / Clin Invest. 2018;128(8):3568-3582.

https://doi.org/10.1172/JCI99888. inflammatory pain at doses that are much lower than those for morphine (7-9). In particular, protectin D1 (PD1) or neuroprotectin D1 (NPD1) is synthesized from docosahexaenoic acid (DHA) and induces potent inhibition of inflammation and inflammatory pain via its multiple actions on neurons, immune cells, and glial cells $(10,11)$. SPMs are known to activate GPCRs to meditate their antiinflammatory and proresolution actions $(8,12,13)$, but the receptors for NPD1 are still unknown.

SPMs are known to resolve inflammation by promoting phagocytosis to remove pathogens and cell debris after inflammation (14). Phagocytosis in mammalian immune cells is activated by the attachment of these cells to pathogen-associated molecular patterns such as zymosan. MФs play a critical role in phagocytosis (15, 16) and exhibit different phenotypes, such as proinflammatory M1-like phenotypes and antiinflammatory M2 phenotypes, which mediate detrimental and protective actions of МФs, respectively (16). However, the signaling mechanisms underlying МФ phagocytosis and phenotypic changes are not fully understood.

GPR37, also known as parkin-associated endothelin-like receptor (Pael-R), is highly expressed in the brain $(17,18)$ and has been implicated in neurological disorders such as Parkinson's disease and autism. GPR37 is a substrate of parkin, and its insoluble aggregates were found to be accumulated in Lewy bodies in brain samples from patients with Parkinson's disease (19). GPR37 also associates with the dopamine transporter to modulate dopamine uptake (20). Mutations in the GPR37 gene are associated with autism spectrum disorders (21). GPR37 is also expressed by oligodendrocytes in the brain and spinal cord to regulate the differentiation of these glial cells (22). The role of GPR37 in immune cells remains unknown. Prosaposin and prosaposin-derived 14-mer peptide (TX14) are ligands of GPR37 and exhibit neuroprotec- 
tive and analgesic effects $(23,24)$. In this study, we found that GPR37 was expressed by MФs but not microglia. We also identified GPR37 as a potential receptor for NPD1, which is required for NPD1-induced intracellular $\mathrm{Ca}^{2+}\left(\mathrm{iCa}^{2+}\right)$ signaling and phagocytosis in MФs. These actions of NPD1 were abolished in Gpr37deficient MФs. Finally, we demonstrated that GPR37 is critical for $\mathrm{M} \Phi-$ mediated resolution of inflammatory pain.

\section{Results}

GPR37 is expressed by MTs, but not microglia. IHC revealed that GRP37 immunoreactivity (IR) is present in the dermis of hind paw skins of WT mice (Figure 1A). We validated the specificity of the GPR37 antibody by loss of staining in the skin of $\mathrm{Gpr} 37^{-/-}$mice (Figure 1B). ISH consistently showed that Gpr37 mRNA was coexpressed with GPR37 protein in the dermis (Supplemental Figure $1 \mathrm{~A}$; supplemental material available online with this article; https:// doi.org/10.1172/JCI99888DS1). Double staining showed that GPR37 was largely colocalized with the MФ marker CD68 (Figure 1A). Flow cytometry showed that GPR37 was expressed in $30 \%$ of F4/80+ MФs (Figure 1, C and D). Thus, GPR37 was primarily expressed by skin MФs (sMФs). We also collected peritoneal MФs (pMФs) for flow cytometric analysis, and the result showed GPR37 expression in 65\% of WT mouse pMФs, but GPR37 expression was absent in $\mathrm{Gpr} 37^{-/-}$mice (Figure 1D). Interestingly, we found that the number of F4/80+ $\mathrm{sM \Phi s} \mathrm{and} \mathrm{pM} \Phi$ s was comparable in WT and Gpr37/- mice (Figure 1D), suggesting that GPR37 does not regulate the genesis or proliferation of MФs. Immunocytochemistry in pMФs showed high levels of colocalization of GPR37 and F4/80 in WT but not Gpr37-/- mice (Figure 1E). Confocal analysis revealed surface and cytoplasm localization of GPR37 in pMФs (Figure 1F). We were also able to detect surface expression of GPR37 by flow cytometry using a non-cell-permeable method (Figure 1, C and D).

Since GPR37 plays a role in neurological and neuropsychiatric diseases (19), we next examined the expression of GPR37 in the PNS (dorsal root ganglia [DRG]) and the CNS (spinal cord and brain). IHC showed GPR37 expression in F4/80 ${ }^{+}$MФs in DRG (Supplemental Figure 1B). Western blotting detected a single band of GRP37 in brain and spinal cord tissues of WT mice, but this band disappeared in $\mathrm{KO}$ mice (Figure $1 \mathrm{G}$ and Supplemental Figure $1 \mathrm{E}$ ). We also detected GPR37 bands in skin and pMФs (Figure $1 \mathrm{G}$ and Supplemental Figure 1E).

Microglia are MФ-like cells in the CNS that release proinflammatory cytokines to regulate neuroinflammation and pathological pain (25). Double staining revealed no colocalization of GPR37 with the microglial marker CX3CR1 in the spinal cords of $\mathrm{Cx} 3 \mathrm{cr} 1-$ GFP mice (Figure 1H). We also used LacZ staining to check for Gpr37 mRNA expression in the spinal cords and brains of Gpr37+/mice. LacZ-labeled nuclei were primarily found in the white matter of the brain (e.g., corpus callosum) (Figure 1I) and the spinal cord (Supplemental Figure 1, C and D). We did not observe colocalization of Gpr37-expressing nuclei (labeled by lacZ) with IBA1, a marker for microglia (Figure 1I). Collectively, these data suggest that GPR37 is expressed by MФs but not microglia.

NPD1 induces $\mathrm{iCa}^{2+}$ increases in HEK293 cells and MФs via GPR37. In order to investigate how GPR37 regulates intracellular signaling, we performed $\mathrm{Ca}^{2+}$ imaging of HEK293 cells that overexpress GPR37. Interestingly, prosaposin and TX14 are ligands of
GPR37 and exhibit similar neuroprotective as analgesic effects (23, 26) as NPD1 (11). Thus, we hypothesized that TX14 and SPMs may activate the same GPCR and elicit similar intracellular responses. We transfected HEK293 cells with human GPR37 cDNA, loaded these cells with the $\mathrm{Ca}^{2+}$ dye Fura-2AM, and stimulated them with TX14 and various SPMs (Figure 2, A-C). TX14 $(1 \mu \mathrm{M})$ and NPD1 $(30 \mathrm{nM})$ elicited significant increases in $\mathrm{iCa}^{2+}(P<0.05)$. The SPMs resolvins (RvE1, RvD1, RvD2) and lipoxin A1, even at a higher concentration (100 nM), failed to increase $\mathrm{iCa}^{2+}$ in GPR37-expressing HEK293 cells. The precursors of these SPMs, DHA and eicosapentaenoic acid (EPA), at a very high concentration $(1 \mu \mathrm{M})$, also did not evoke calcium responses (Figure $2 \mathrm{C}$ ). Dose-response analysis revealed that NPD1 is more potent than TX14 in inducing $\mathrm{iCa}^{2+}$ increases in HEK293 cells (Figure 2D), with respective $\mathrm{EC}_{50}$ of 26 $\mathrm{nM}$ and $407 \mathrm{nM}$ for NPD1 and TX14. We found that the NPD1induced $\mathrm{iCa}^{2+}$ increase was completely blocked by pertussis toxin (PTX), indicating the involvement of Gi-mediated GPCR signaling (Figure 2E). The NPD1-induced $\mathrm{iCa}^{2+}$ increase was also inhibited by thapsigargin, BAPTA-AM [1,2-bis(o-aminophenoxy) ethane- $N, N, N^{\prime}, N^{\prime}$-tetraacetic acid acetoxymethy ester], and EGTA (Figure 2E), suggesting that both influx of extracellular $\mathrm{Ca}^{2+}$ and release from the $\mathrm{iCa}^{2+}$ store contribute to the increase in $\mathrm{iCa}^{2+}$.

Several SPM receptors, such as CMKLR1 (also known as ChemR23) for RvE1, GPR32 for RvD1, and ALX, have been identified for lipoxin A $(7,12,13)$. We transfected human cDNAs of CMKLR1, GPR32, and ALX into HEK293 cells and checked $\mathrm{Ca}^{2+}$ responses following the treatment with each SPM. Notably, NPD1 failed to elicit an $\mathrm{iCa}^{2+}$ increase in HEK293 cells expressing ChemR23, GPR32, and $A L X$ (Supplemental Figure 2, A-C), indicating a specific action of NPD1 on GPR37. RvE1, RvD1, and lipoxin A also failed to evoke $\mathrm{Ca}^{2+}$ responses in HEK293 cells that expressed CHEMR23, GPR32, and ALX, respectively (Supplemental Figure $2, \mathrm{~A}-\mathrm{C})$. This result suggests that, compared with these SPMs, the $\mathrm{Ca}^{2+}$ response is unique to NPD1.

To determine whether NPD1 and TX14 bind to GPR37, we conducted a dot blot assay by coating the blots with different concentrations of NPD1 and TX14. We also included RvE1 as a negative control. The blots were then incubated with cell lysates from HEK293 cells transfected with GPR37 cDNA or empty vector (mock transfection). We found a dose-dependent binding of TX14 and NPD1, but not RvE1, to GPR37-containing lysates, whereas cell lysates from the mock transfection (GPR37-negative) did not show binding to TX14 or NPD1 (Figure 2F, Supplemental Figure 3, A-C). Dose-response curve analysis revealed that NPD1 has a higher binding affinity for GPR37 than for TX14 (Supplemental Figure 3B).

Next, we conducted GPR37-mediated $\mathrm{Ca}^{2+}$ imaging in pMФs from WT and $\mathrm{Gpr} 37^{-/-}$mice using Fluo-4AM as a $\mathrm{Ca}^{2+}$ indicator, since this dye works better than Fura-2AM for pMФs. We found that NPD1 (30 nM) elicited a robust and sustained $\mathrm{iCa}^{2+}$ increase in $\mathrm{pM} \Phi$ s but that this increase was abolished in Gpr37-deficient pMФs (Figure 2, G and H). As compared with the $\mathrm{iCa}^{2+}$ increase in HEK293 cells, NPD1 triggered a slow-onset but more persistent $\mathrm{Ca}^{2+}$ response in pMФs (Figure 2, B and G). A relatively rapid calcium response in HEK293 cells (reaching a peak in 2 to $3 \mathrm{~min}$ ) may result from overexpression of GRP37. A delayed and sustained $\mathrm{iCa}^{2+}$ increase in $\mathrm{PM} \Phi$ s (reaching a peak in 7 to $10 \mathrm{~min}$ ) may result 
A
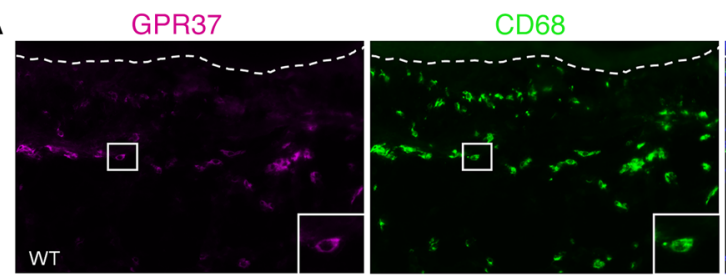

GPR37 CD68 DAPI
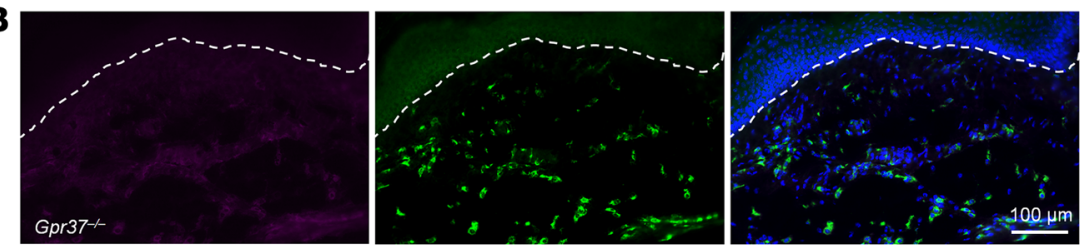

C
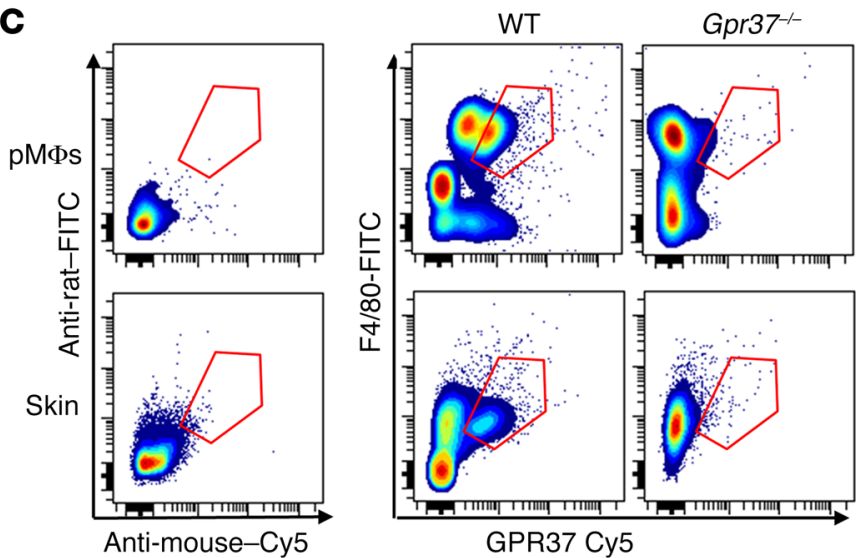

D

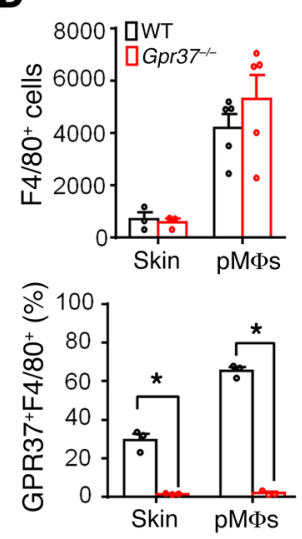

E

GPR37

GPR37 F4/80

GPR37 F4/80
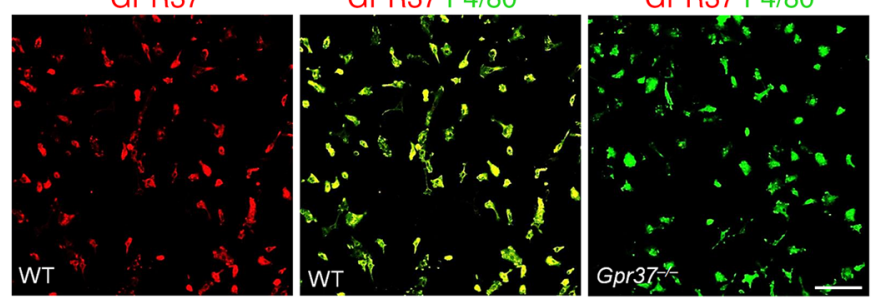

F

GPR37

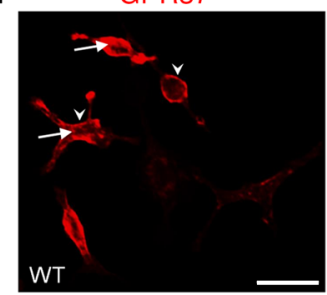

G

H
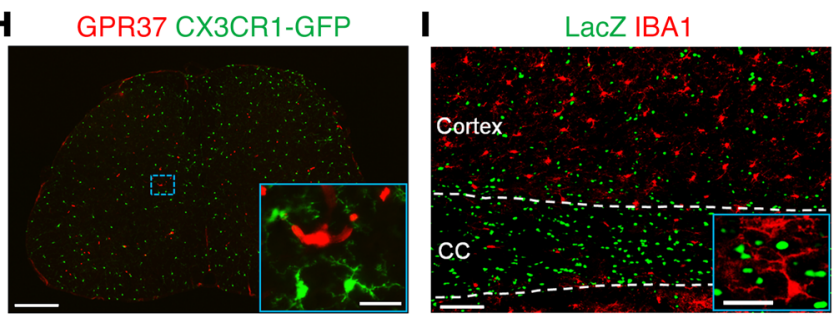

Figure 1. GPR37 is expressed by MФs, not microglia. (A and B) IHC showing the colocalization of GRP37 and CD68 in the hind paw dermis of WT (A) but not Gpr37-1- (B) mice. Boxes in A indicate an enlarged cell. Blue DAPI staining labels all nuclei in the skin. Scale bar: $100 \mu \mathrm{m} ; 45 \mu \mathrm{m}$ (original scale in inset). (C and D) Flow cytometry showing GPR37 expression in F4/80+ MФs from hind paw skin and pMФs from WT and KO (Gpr37-1-) mice. $n=3-5$ mice/group. Data represent the mean $\pm \mathrm{SEM}$. ${ }^{*} P<0.05$; unpaired $t$ test. (E) Double staining showing GPR37 IR in F4/80 ${ }^{+}$pMФs. Scale bar: $100 \mu \mathrm{m}$. (F) Confocal microscopic images showing cytoplasm (arrows) and surface (arrowheads) localization of GPR37 IR in pMФs. Scale bar: $20 \mu \mathrm{m}$. (G) Western blot showing a single band of GRP37 in lysates of brain, spinal cord (SC), hind paw skin, and pMФs. Note that the band is absent in Gpr37-l- mice. (H) Double staining showing no colocalization of GPR37 with CX3CR1 in the spinal cords of CX3cr1-GFP mice. Scale bars: $250 \mu \mathrm{m}$ and $20 \mu \mathrm{m}$ (inset). (I) $\beta$-Cal staining showing no colocalization of LacZ (Gpr37) expression with IBA1 in brain sections from Gpr37 $7^{+-}$mice. Scale bars: $100 \mu \mathrm{m}$ and $20 \mu \mathrm{m}$ (inset). CC, corpus callosum. 
A
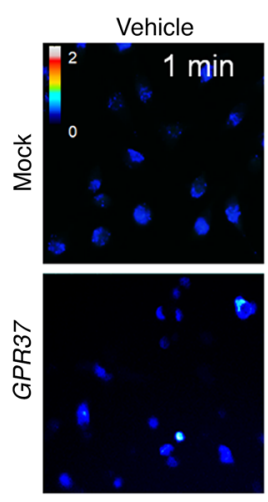

HEK293 cells

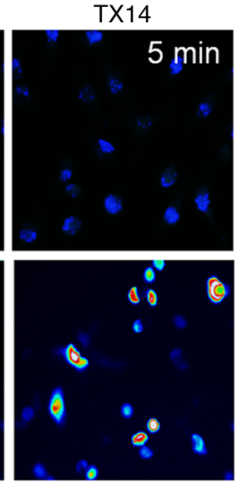

B

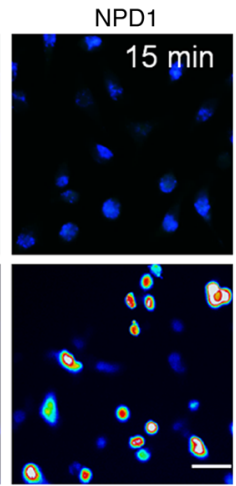

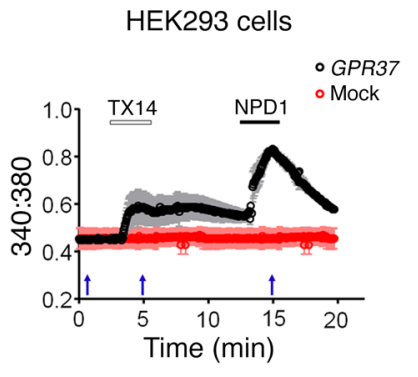

C

HEK293 cells

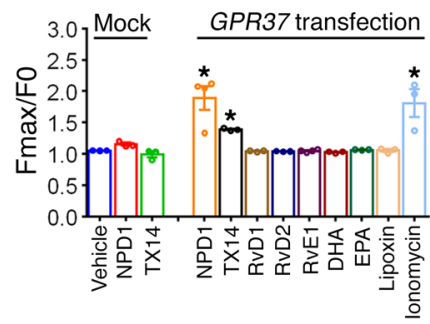

D
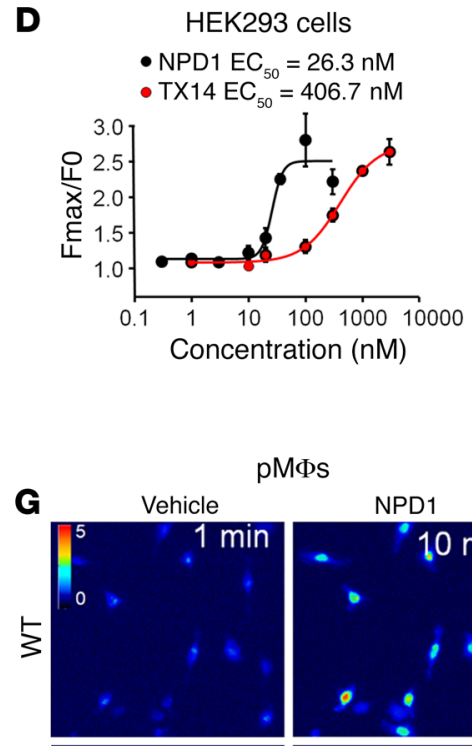

pMథs

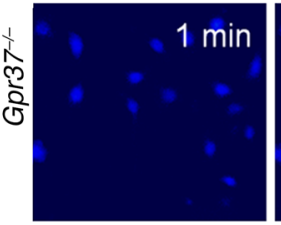

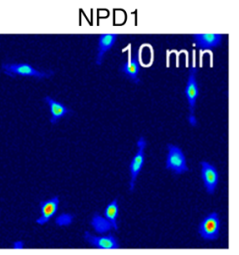

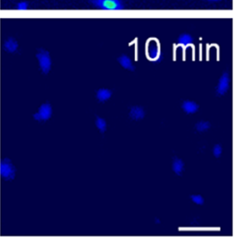

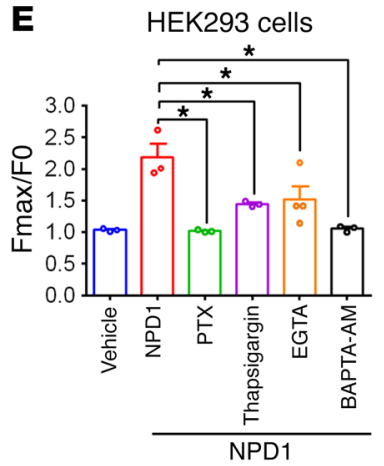

$\mathbf{F}$

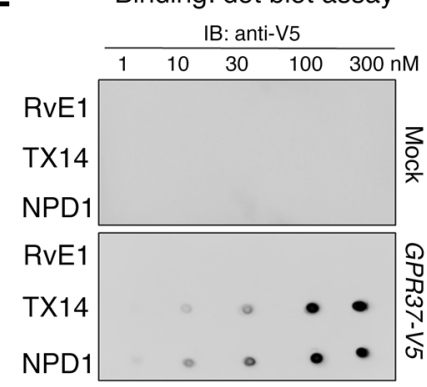

H $\quad \mathrm{pM} \Phi \mathrm{s}$

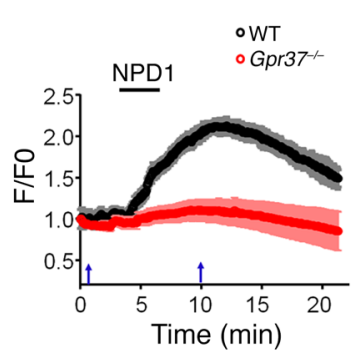

I

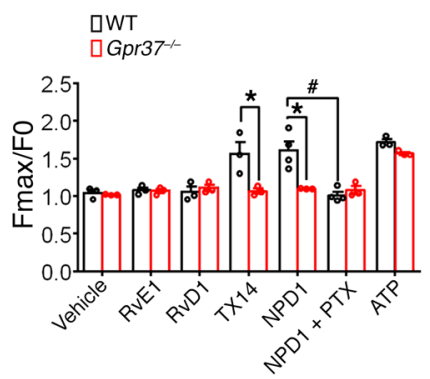

$\mathbf{J}$
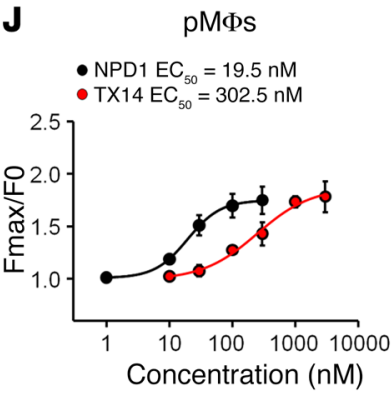

Figure 2. NPD1 induces iCa ${ }^{2+}$ increases in HEK293 cells and MФs via GPR37. (A-E) Ca ${ }^{2+}$ imaging with the Fura-2AM indicator in HEK293 cells transfected with GPR37 cDNA or empty vector (mock transfection). (A) Representative images showing calcium responses (color changes) after TX14 (1 $\mu$ M, 3 minute treatment) and NPD1 ( $30 \mathrm{nM}, 3$ minute [duration of treatment]) treatment. Scale bar: $50 \mu \mathrm{m}$. The pseudo-color scale (0-2) shows the possible range of calcium signaling. (B) Combined traces from 100 cells showing time-dependent iC $\mathrm{a}^{2+}$ increases induced by NPD1 and TX14 after GPR37 but not mock transfection. Arrows show the time points at which the images in A were collected. (C) Comparison of iCa ${ }^{2+}$ levels after 3 minutes of treatment with NPD1 (30 nM), TX14 (1 $\mu$ M), RvD1 (100 nM), RvD2 (100 nM), RvE1 (100 nM), lipoxin (100 nM), DHA (1 $\mu$ M), EPA $(1 \mu M)$, and ionomycin $(2 \mu M) .{ }^{*} P<0.05$ versus baseline (vehicle); 2-way ANOVA followed by Bonferroni's post hoc test. $n=3-4$ cultures, with 73 to 340 cells analyzed for each condition. (D) Dose-response curves of NPD1- and TX14-induced iC $a^{2+}$ increases. $n=3-4$ cultures, with 72 to 250 cells analyzed for each condition. Note the different $\mathrm{EC}_{50}$ values for these 2 compounds. (E) Inhibition of NPD1-induced ( $30 \mathrm{nM})$ iCa ${ }^{2+}$ increases by PTX ( $1 \mu \mathrm{g} / \mathrm{ml}, 16 \mathrm{~h}$ before treatment), thapsigargin ( $\left.1 \mu \mathrm{M}, 3 \mathrm{~min}\right)$, and EGTA (10 mM, 3 min). ${ }^{*} P<0.05$; 1 -way ANOVA. $n=3-4$ cultures, with 131 to 186 cells analyzed per treatment. (F) Dot blots showing a dose-dependent binding of NPD1 and TX14, but not RvE1, to GPR37. The blots were coated with NPD1, TX14, and RVE1 and then incubated with cell lysates from HEK293 cells with GPR37 cDNA or mock transfection. (G-J) $\mathrm{Ca}^{2+}$ imaging with the Fluo-4AM $\mathrm{Ca}^{2+}$ indicator in WT and Gpr37-1- pMФ cultures. (G) Representative images showing $\mathrm{Ca}^{2+}$ responses (color changes) after NPD1 treatment (30 nM) in WT but not Gpr37-1- mice. Pseudo-color scale (0-5) shows the possible range of calcium signaling. Scale bar: $50 \mu \mathrm{m}$. (H) Combined traces from 100 cells showing time-dependent iCa ${ }^{2+}$ responses after NPD1 treatment (100 nM, 3 min) in WT and

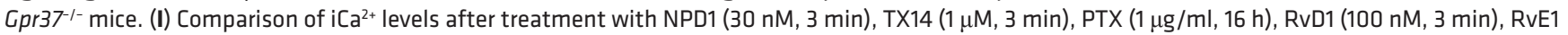

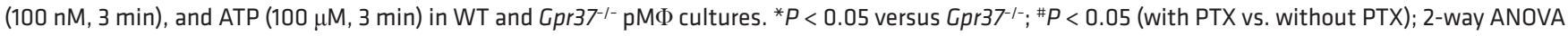
followed by Bonferroni's post hoc test. $n=3-4$ cultures, with more than 300 cells analyzed for each condition. (J) Dose-response curves of NPD1- and TX14-induced iCa ${ }^{2+}$ increases in PMФ cultures. $n=3$ cultures, with 80-500 cells analyzed for each condition. Note the different EC ${ }_{50}$ values for NPD1 and TX14. Data represent the mean \pm SEM. F0, relative basal intensity; Fmax, relative peak intensity. 
A
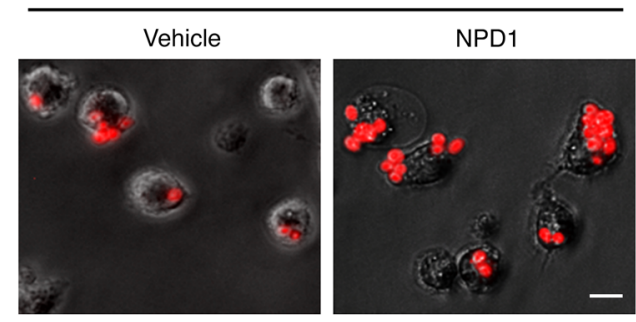

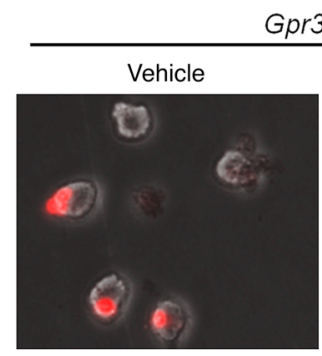

Gpr37-1-

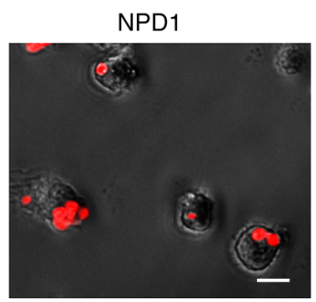

B

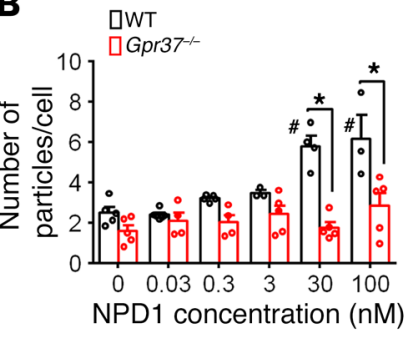

C
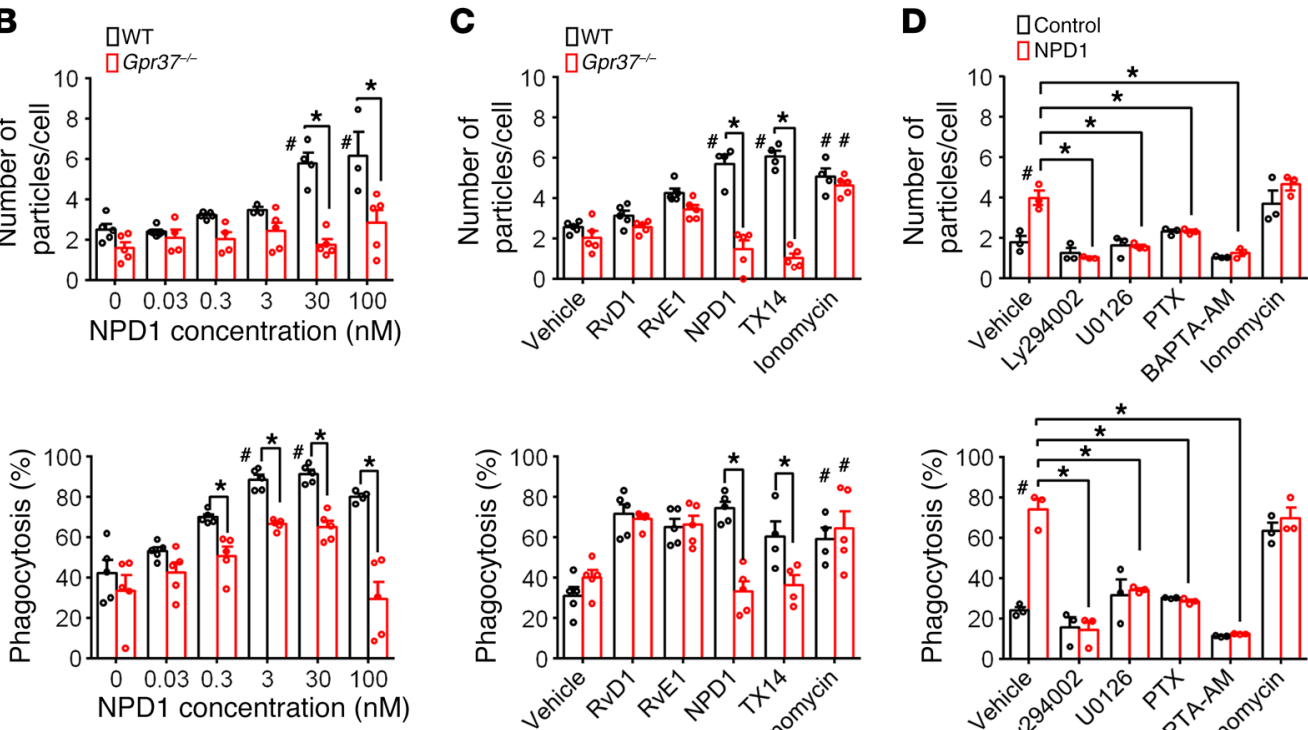
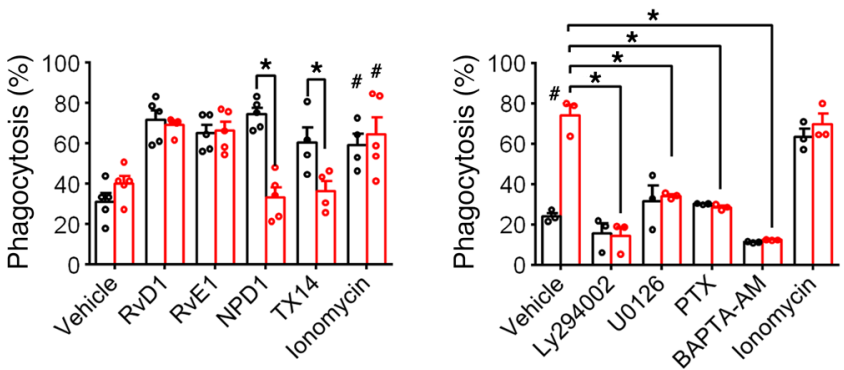

Figure 3. NPD1 enhances MФ phagocytic activity in vitro via GPR37. (A) NPD1 enhanced phagocytosis in WT pMФs, as revealed by fluorescence-labeled

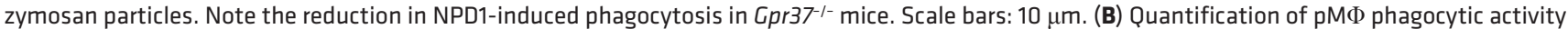
according to the number of zymosan particles (top) and percentage of cells (bottom) with phagocytic activity (>1 particle/cell). Note the dose-dependent phagocytic activity induced by NPD1. ${ }^{\#} P<0.05$ versus control (vehicle, PBS); ${ }^{*} P<0.05$ versus Gpr37-I-; 2 -way ANOVA followed by Bonferroni's post hoc test. $n=4-5$ cultures/group. (C) Phagocytic activity in pMФs from WT and Gpr37-1- mice following treatment with RvD1 (100 nM), RvE1 (100 nM), TX14 (100 $\mathrm{nM}$ ), and ionomycin $(2 \mu \mathrm{M})$, as revealed by the number of zymosan particles (top) and percentage of cells with phagocytosis (bottom). ${ }^{\#} P<0.05$ versus vehicle; ${ }^{*} P<0.05$ versus Gpr37-1-; 2-way ANOVA followed by Bonferroni's post hoc test. $n=3-5$ cultures/group. (D) Effects of LY294002 (50 $\mu M$ ), U0126 (10 $\mu \mathrm{M}), \operatorname{PTX}(1 \mu \mathrm{g} / \mathrm{ml})$, BAPTA-AM $(10 \mu \mathrm{M})$, and ionomycin $(2 \mu \mathrm{M})$ on basal and NPD1-induced (30 nM) phagocytosis. ${ }^{*} P<0.05$ versus vehicle (with NPD1); $\# P<0.05$, NPD1 versus control; 2-way ANOVA followed by Bonferroni's post hoc test. $n=3-5$ cultures/group. For each culture, $113-503$ cells were analyzed. Data represent the mean $\pm \mathrm{SEM}$.

from the expression of additional ion channels such as transient receptor potential (TRP) channels in these immune cells. It is noteworthy that MФs express TRPV2 (27) and TRPM2 $(28,29)$, which are involved in phagocytosis during bacterial infection. Like NPD1, TX14 also elicited a GPR37-dependent $\mathrm{iCa}^{2+}$ increase in pMФs (Figure 2H). As expected, RvD1 and RvE1 (100 nM) did not alter $\mathrm{iCa}^{2+}$ levels in pMФs (Figure 2I). We found that the NPD1triggered $\mathrm{iCa}^{2+}$ increase in $\mathrm{pM} \Phi$ s was also $\mathrm{Gi}$ dependent, since PTX blocked this increase (Figure 2I). For comparison, we observed that the ATP-evoked $\mathrm{iCa}^{2+}$ increase was not altered in GPR37deficient $\mathrm{pM \Phi s} \mathrm{(Figure} \mathrm{2I).} \mathrm{Dose-response} \mathrm{analysis} \mathrm{showed} \mathrm{that}$ NPD1 was more potent than TX14 in inducing $\mathrm{iCa}^{2+}$ increases in pMФs, with respective $\mathrm{EC}_{50}$ values of $19.5 \mathrm{nM}$ and $302.5 \mathrm{nM}$ for NPD1 and TX14 (Figure 2J). Together, these data suggest that GPR37 serves as a receptor for NPD1 and mediates NPD1-elicited $\mathrm{Ca}^{2+}$ responses in both heterologous cells and native $\mathrm{pM \Phi s.} \mathrm{There}$ was no obvious desensitization of $\mathrm{Ca}^{2+}$ signaling after repeated applications of NPD1 or TX14 in pMФs (Supplemental Figure 2,
$\mathrm{D}$ and $\mathrm{E})$. The percentages of MФs that responded to NPD1 and TX14 were almost identical (Supplemental Figure 2F).

NPD1 increases $M \Phi$ phagocytosis in vitro via GPR37. We investigated whether $\mathrm{iCa}^{2+}$ increases would trigger phagocytosis of pathogens such as zymosan by МФs (30). As a widely used pathogen, zymosan activates TLR2 to trigger inflammation and inflammatory pain in rodents after intraplantar (i.pl.) injection $(31,32)$. We tested phagocytosis in $\mathrm{pM \Phi s} \mathrm{using} \mathrm{fluorescence-labeled} \mathrm{zymosan} \mathrm{parti-}$ cles. NPD1 caused a dose-dependent increase in phagocytic activity; we observed more zymosan particles in $\mathrm{PM \Phi s} \mathrm{after} \mathrm{the} \mathrm{treatment}$ (30 nM for $30 \mathrm{~min}$ ) (Figure 3, A and B). NPD1 further increased the percentage of $\mathrm{pM} \Phi$ s showing the phagocytosis of zymosan particles (Figure 3B). These NPD1-evoked increases in phagocytic activity were dose dependent in WT МФs and abolished in Gpr37-deficient MФs (Figure 3, A and B). МФ phagocytosis was also induced by TX14, RvD1, and RvE1, but only the TX14-induced phagocytosis required Gpr37 (Figure 3C). However, the basal phagocytic activity did not change in pMФs from Gpr37-KO mice (Figure 3C). 
We further explored the intracellular signaling mechanisms underlying phagocytosis in pMФs. NPD1-induced phagocytosis, as well as baseline phagocytosis, was blocked by BAPTA-AM, indicating a critical involvement of $\mathrm{iCa}^{2+}$ (Figure 3D). By contrast, ionomycin $(2 \mu \mathrm{M})$ markedly increased phagocytosis in both WT and Gpr37-KO mice (Figure 3C), suggesting that $\mathrm{iCa}^{2+}$ is sufficient to trigger phagocytosis. NPD1-induced phagocytosis was also inhibited by the Gi blocker PTX, the ERK pathway inhibitor U0126, and the PI3K/AKT pathway inhibitor LY294002 (Figure 3D). Collectively, our results suggest that NPD1 induces phagocytosis of $\mathrm{pM \Phi s}$ via GPR37, $\mathrm{iCa}^{2+}$, and the PI3K/AKT and ERK signaling pathways.

GPR37 regulates $M \Phi$ phagocytosis in inflamed hind paw skin. We took advantage of pHrodo Red dye-conjugated zymosan, which shows red fluorescence after phagocytosis, because of the lower $\mathrm{pH}$ values in intracellular compartments such as phagosomes $(\mathrm{pH}$ 5-6) (Figure 4A). Using the zymosan model, we compared the time course of edema, infiltration of immune cells (neutrophils and $\mathrm{M \Phi s),} \mathrm{and} \mathrm{phagocytosis} \mathrm{of} \mathrm{zymosan} \mathrm{particles} \mathrm{and} \mathrm{neutrophils} \mathrm{by}$ MФs following i.pl. injection of the labeled zymosan particles (20 $\mu \mathrm{g} / 20 \mu \mathrm{l})$ into a hind paw (Figure $4 \mathrm{~B}$ ). Zymosan produced rapid edema, as indicated by an increase in hind paw volume. This edema was evident at 2 hours, peaked at 4 hours, declined but was maintained on day 1 , and recovered on day 8 (Figure 4C). IHC revealed a sequential infiltration of immune cells, first neutrophils $\left(\mathrm{Gr}-1^{+}\right)$, then MФs $\left(\mathrm{CD} 68^{+}\right)$, into the inflamed hind paw skin (Figure $4 \mathrm{D})$. Gr-1 IR began to increase at 4 hours, peaked at 24 hours, and declined on day 2 (Figure 4D and Supplemental Figure 4A). CD68 IR showed a delayed but sustained increase in inflamed paw skin that began on day 1 , peaked on day 5 , and declined but remained elevated on days 8 and 21 (Figure 4D and Supplemental Figure 4A). The time courses of zymosan phagocytosis and GPR37 IR were very similar to that of CD68 IR in sMФs, peaking on day 5 (Figure 4, D and E, Supplemental Figure 3A). Double immunostaining demonstrated that (a) GRP37 was expressed in $\mathrm{CD}^{+} 8^{+}$MФs (Supplemental Figure 4B), and (b) phagocytized zymosan particles predominantly localized in SMФs expressing GPR37 in the inflamed paws of WT mice (Figure 4F). However, phagocytized zymosan levels in the inflamed hind paw skin were markedly reduced in Gpr37-KO mice (Figure 4G and Supplemental Figure 5A). As compared with WT mice, Gpr37-KO mice did not show any changes in the intensity of CD68 IR in the skin of naive mice or inflamed mice on day 1 or day 5 (Figure $4 \mathrm{H}$ and Supplemental Figure 5B), but showed a partial reduction in $\mathrm{F} 4 / 80^{+} \mathrm{sM} \Phi$ s in the inflamed skins on day 5 (Supplemental Figure 5C). Thus, a lack of GPR37 has limited impact on the general population of $\mathrm{M} \Phi$ s, especially during the induction phase of inflammation. Notably, we observed a delayed increase in GPR $37^{+}$sMФs in the inflamed paws in WT mice, with a peak on day 5 (Figure 4I and Supplemental Figure 5D). This result suggests a role of GPR37-expressing sMФs in the resolution of inflammation.

Phagocytic activity against apoptotic neutrophils is also critical for the resolution of inflammation (33). In agreement with the IHC analysis, flow cytometry confirmed a time-dependent change in neutrophil numbers after zymosan injection: the number of skin neutrophils peaked on day 1 but returned to baseline on day 5 in WT mice, indicating an elimination of neutrophils during the resolution phase (Supplemental Figure 6A). Compared with WT mice, we found that neutrophil numbers in the inflamed skin of $G$ pr37-/- mice were unaltered $(P>0.05$, Supplemental Figure 6A). Importantly, the phagocytic activity of sMФs against neutrophils was

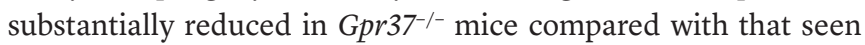
in WT mice (Supplemental Figure 6B). Histochemical examination also showed sMФs engulfing Gr-1-labeled neutrophils in the inflamed skin of WT mice (Supplemental Figure 6, C and D). Taken together, these findings indicate that zymosan induces distinct time courses in edema, neutrophil infiltration, and $М \Phi$ infiltration in the inflamed skin and that GPR37 plays an essential role in MФ phagocytosis of pathogens (zymosan particles) and apoptotic neutrophils in the inflamed skin.

GPR37 regulates distinct expression of pro- and antiinflamma-

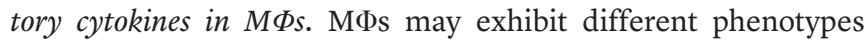
such as the resting phenotype (M0), proinflammatory phenotypes (M1-like), and antiinflammatory/proresolution phenotypes (M2-like) (34). These phenotypes are characterized by distinct expression of proinflammatory cytokines (e.g., IL-1 $\beta$ ) and antiinflammatory cytokines (IL-10, TGF- $\beta$ ). Zymosan increased both Il1b mRNA levels and IL-1 $\beta$ protein levels in the inflamed skin of WT mice, but these increases were greater in Gpr37-KO mice (Figure 5, A and B). Zymosan also increased the expression of the antiinflammatory cytokines IL-10 and TGF- $\beta$ at both mRNA and protein levels in inflamed skin. Notably, these increases were abolished in the $\mathrm{KO}$ mice, especially in the resolution phase of inflammation on day 5 (Figure $5, \mathrm{C}-\mathrm{F}$ ). In pMФ cultures, NPD1 suppressed IL-1 $\beta$ levels but increased IL-10 and TGF- $\beta$ levels following zymosan treatment, and these actions of NPD1 required GPR37 (Supplemental Figure 7). Therefore, GPR37 regulated M $\Phi$ phenotypes by suppressing the expression of proinflammatory cytokines (IL-1 $\beta$ ) and increasing the expression of antiinflammatory cytokines (IL-10 and TGF- $\beta$ ) in zymosan-activated MФs in the inflamed skin.

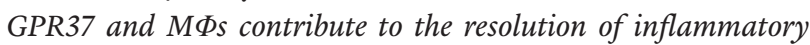
pain. We investigated whether zymosan-induced inflammatory pain would be altered in $G p r 37^{-/-}$mice by conducting a timecourse study of inflammatory pain from 2 hours to 28 days (Figure $6 \mathrm{~A})$. Administration of i.pl. zymosan elicited rapid ( $<2 \mathrm{~h})$ inflammatory pain in WT mice, as characterized by heat hyperalgesia and observed by a reduction in paw withdrawal latency to radiant heat stimulation, and mechanical allodynia, as observed by a reduction in the paw withdrawal threshold to von Frey hair stimulation. Mechanical allodynia was more persistent than heat hyperalgesia in WT mice, resolving on day 5 and day 2, respectively (Figure 6, A and B). Compared with WT mice, zymosan-induced heat hyperalgesia and mechanical allodynia were prolonged in $\mathrm{Gpr} 37^{-/-}$mice. The duration of heat hyperalgesia was prolonged from 2 days in WT mice to 8 days in $\mathrm{KO}$ mice (Figure 6B). In particular, the duration of mechanical allodynia was prolonged from 5 days in WT mice to more than 28 days in $\mathrm{KO}$ mice. Indeed, mechanical allodynia failed to resolve in Gpr37 $7^{-/-}$mice by day 28 (Figure 6B). IL-1 $\beta$ is a key proinflammatory cytokine and critically involved in inflammatory pain (6). Injection (i.pl.) of IL-1 $\beta$ induced heat hyperalgesia and mechanical allodynia in WT mice, but both pain symptoms were prolonged in $\mathrm{Fpr}^{37^{-/}}$mice (Figure 6C).

We also compared baseline pain sensitivity in WT and KO mice by assessing heat sensitivity in Hargreaves and hot plate tests; mechanical sensitivity in von Frey, Randall-Selitto, and pinprick tests; and cold sensitivity in acetone tests. We did not observe 
A pHrodo Red dye conjugated zymosan

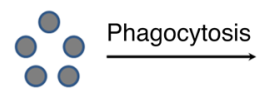

Nonfluorescent outside the cell $(\mathrm{pH} 7)$

C

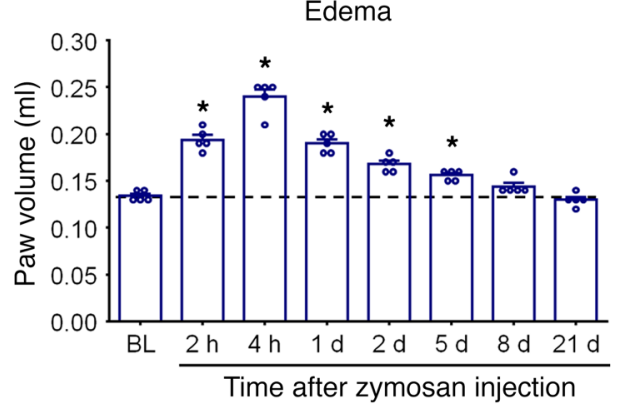

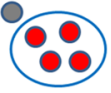

Red fluorescence in phagosomes $(\mathrm{pH} \mathrm{5-6)}$
B

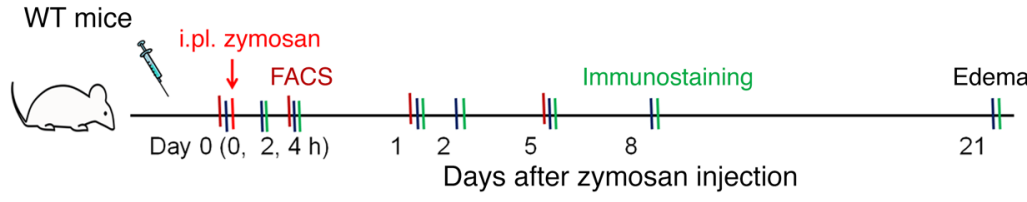

E
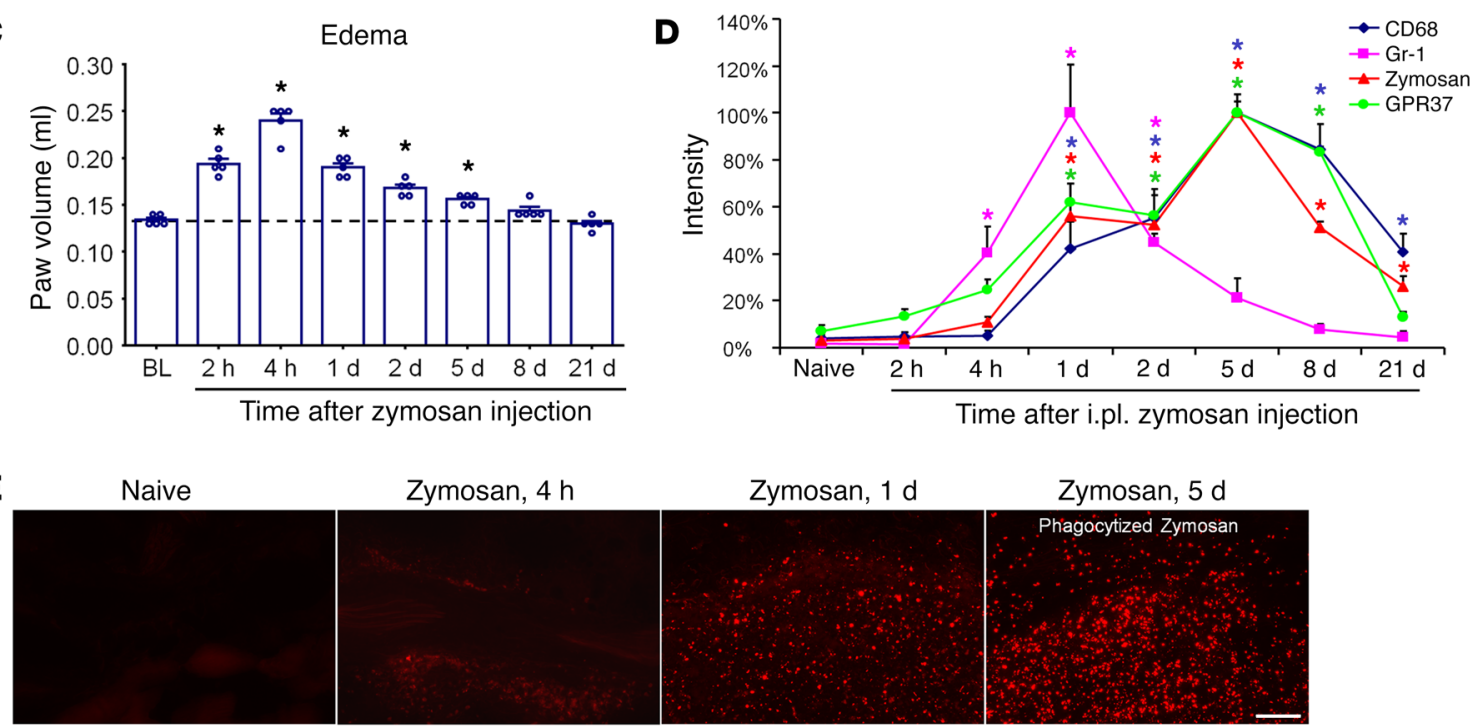

F
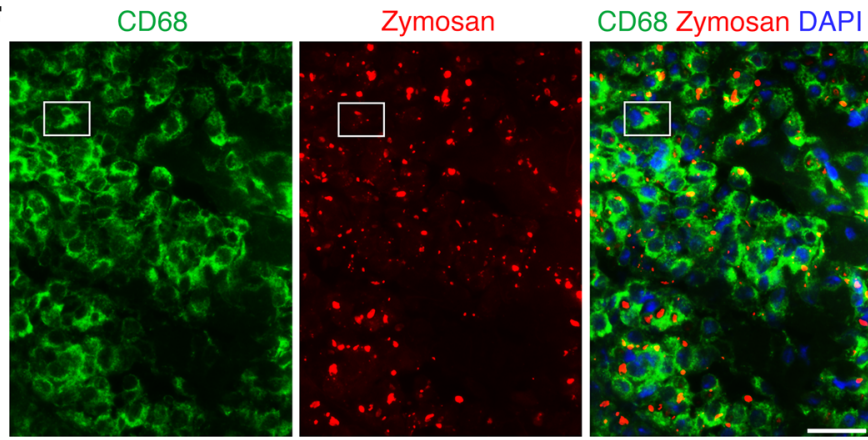

G

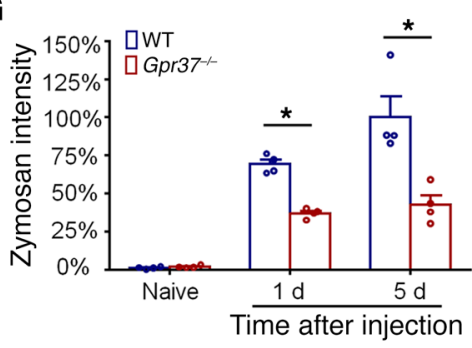

H

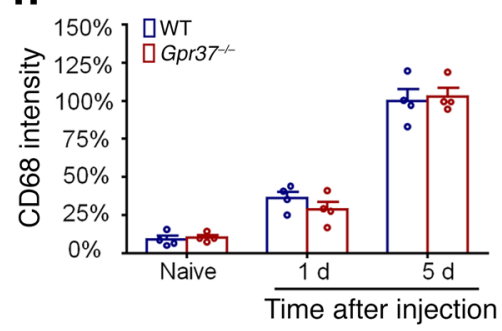

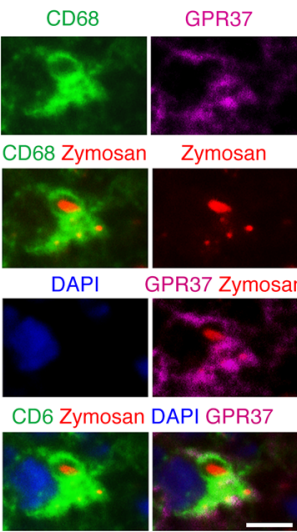

Zymosan, $5 \mathrm{~d}$

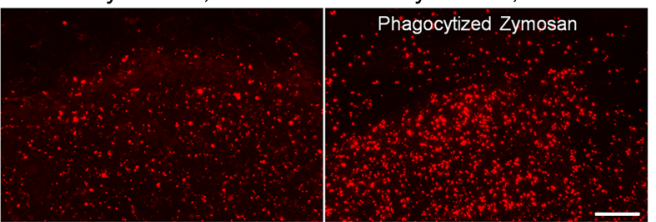

Zymosan, $1 \mathrm{~d}$

I

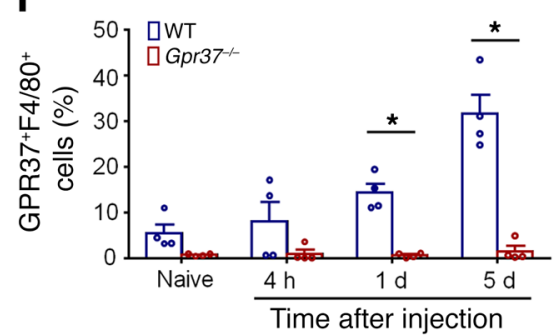

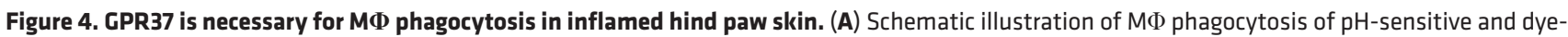
conjugated zymosan (pH-R-zymosan) particles. Note that only phagocytized zymosan particles show red fluorescence. (B) Experimental diagram showing the timeline of i.pl. injection of pH-R-zymosan, FACS analysis, immunostaining, and edema tests. (C) Edema in a hind paw following zymosan (20 $\mu \mathrm{g} / 20 \mu \mathrm{l}$ ) injection, as measured by paw volume. ${ }^{*} P<0.05$ versus baseline (BL); 1 -way ANOVA. $n=5$ mice/group. (D) IHC showing the time courses of zymosaninduced changes in neutrophils ( $\left.\mathrm{Gr}-1^{+}\right), \mathrm{M} \Phi \mathrm{s}\left(\mathrm{CD68}{ }^{+}\right)$, GPR37, and phagocytized zymosan in inflamed hind paw skins. ${ }^{*} P<0.05$ versus baseline in naive animals; 1-way ANOVA. $n=4$ mice/group. (E) Images of phagocytized zymosan particles in skins of naive mice and inflamed mice 4 hours, 1 day, and 5 days after zymosan injection. Scale bar: $50 \mu \mathrm{m}$. (F) Quadruple staining of CD68 (green), DAPI (blue), GRP37 (purple), and zymosan particles (red) in inflamed skin 5 days after zymosan injection. Note that phagocytized zymosan particles are present inside GPR37+ MФs. Scale bars: $20 \mu \mathrm{m}$ and $5 \mu \mathrm{m}$ (enlarged images). (C) Phagocytized zymosan levels (revealed by staining intensity) in naive and inflamed paws of WT and Gpr37-1- mice. ${ }^{*} P<0.05 ; 2$-way ANOVA. $n=4$ mice/ group. (H) Quantification of CD68 IR in hind paw skin. $n=4$ mice/group. (I) Flow cytometry showing the percentage of GPR37-expressing MФs in WT and Gpr37-/- mice at different time points of zymosan injection. ${ }^{*} P<0.05$; Student's $t$ test. $n=4-5$ mice/group. Data represent the mean \pm SEM. 

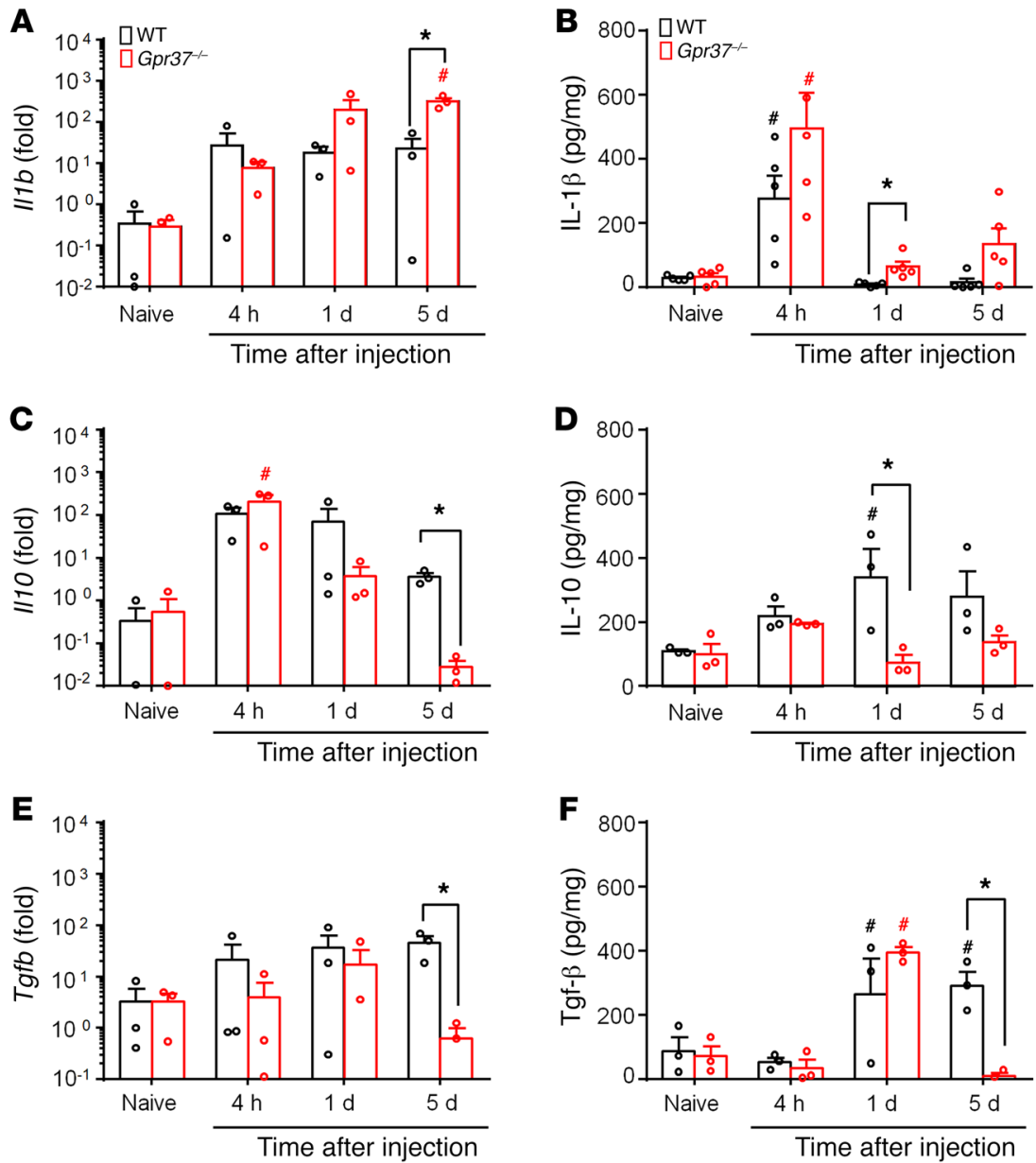

Figure 5. GPR37 is required to regulate cytokine expression in inflamed skin. (A-F) mRNA expression (revealed by quantitative RT-PCR in A, C, and E) and protein expression (revealed by ELISA in B, $\mathbf{D}$, and F) of the proinflammatory cytokine IL-1 $\beta$ (B) and the antiinflammatory cytokines IL-10 (D) and TCF- $\beta$ (F) in noninflamed skins (naive) and inflamed skins of WT and $\mathrm{Gpr} 37^{-1-}$ mice. mRNA expression levels were normalized to Gapdh mRNA. ${ }^{*} P<0.05$ versus Gpr37-1-; ${ }^{\#} P<0.05$ versus naive; 2 -way ANOVA. $n=3-5$ mice/ group. Data represent the mean \pm SEM.

(Figure 7A and Supplemental Figure 9A). In contrast, the number of neutrophils was not affected by the toxin (Supplemental Figure 9B). Importantly, this $\mathrm{M} \Phi$ depletion recapitulated the pain phenotypes observed in $G p r 37^{-/-}$mice: the resolution, but not the induction, of inflammatory pain (heat hyperalgesia and mechanical allodynia) was impaired after the clodronate treatment (Figure 7B). To determine whether МФs are sufficient for the resolution of inflammatory pain, we performed an adoptive transfer of MФs into WT and Gpr37-KO mice 1 day after the zymosan injection. Strikingly, the deficits in inflammatory pain resolution in the KO mice were rescued by the transplantation of WT МФs. Thus, we found no differences in the recovery of heat hyperalgesia or mechanical allodynia between the WT and KO mice (Figure 7, C and D). As expected, adoptive

any changes in these sensory tests in naive $G p r 37^{-/-}$mice that had no tissue injury or inflammation (Figure 6, B and D). It is suggested that the neural pathways of normal pain perception are intact in KO mice. Nor did the KO mice display impairment of motor function in the Rotarod test (Figure 6E). Compared with WT mice, Gpr37-KO mice also had normal development of the sensory system and showed no loss of DRG sensory neurons or their peripheral or central innervations (Supplemental Figure 8). WT and KO mice showed comparable acute inflammatory pain induced by i.pl. injection of capsaicin (Figure 6F). Furthermore, the onset and early development of inflammatory pain, in the first several hours, were unaltered in the zymosan and IL-1 $\beta$ models (Figure $6, \mathrm{~B}$ and C). Zymosan induced identical edema (paw swelling) in WT and KO mice (Figure 6G). Notably, the edema was associated with the induction phase of inflammatory pain, peaking within 4 hours and resolving within 2 days in both WT and KO mice (Figure $6 \mathrm{G})$. Therefore, GPR37 regulates the resolution phase but not the induction phase of inflammatory pain.

Given the important role of GRP37 in MФ signaling, we examined the specific contribution of МФs to zymosan-induced inflammatory pain using both loss-of-function (M $\Phi$ toxin) and gain-of-function (cell adoptive transfer) approaches. Depletion of MФs via systemic injection of the MФ toxin clodronate (35), administered 2 hours and 48 hours prior to the zymosan injection, largely reduced the number of $\mathrm{M} \Phi$ s in the inflamed skin transfer of Gpr37-deficient MФs from KO mice failed to resolve the inflammatory pain deficit in the KO mice (Figure 7E). Collectively, our data suggest that GPR37 in MФs critically contributes to the resolution of inflammatory pain.

IL-10 was implicated in the protection against and resolution of neuropathic pain $(36,37)$. To define a role of IL-10 in the resolution of zymosan-induced inflammatory pain, we administered anti-IL-10-neutralizing antibody or control IgG 1 day after the induction of inflammation (Figure 8A). Compared with control $\mathrm{IgG}$, the neutralizing antibody impaired the resolution of heat hyperalgesia and mechanical allodynia (Figure 8B).

Finally, we investigated whether МФs resolve inflammatory pain via the release of IL-10. To this end, we blocked IL-10 from MФs by i.pl. coadministration of WT MФs and the anti-IL-10 antibody to WT and KO mice (Figure 8C). As shown Figure 7D, we demonstrated that transplantation of WT MФs into Gpr37deficient mice could promote the resolution of inflammatory pain. However, the proresolving effect of WT MФs was compromised by the coadministration of the anti-IL-10 antibody (Figure 8D). This result suggests that WT MФs may contribute to the resolution of inflammatory pain in KO mice via IL-10 secretion.

\section{Discussion}

Phagocytosis is a key function of MФs in the resolution of inflammation. Increasing evidence suggests that SPMs such as resolvins, 
A WT and $G$ pr3 $7^{-1-}$ mice
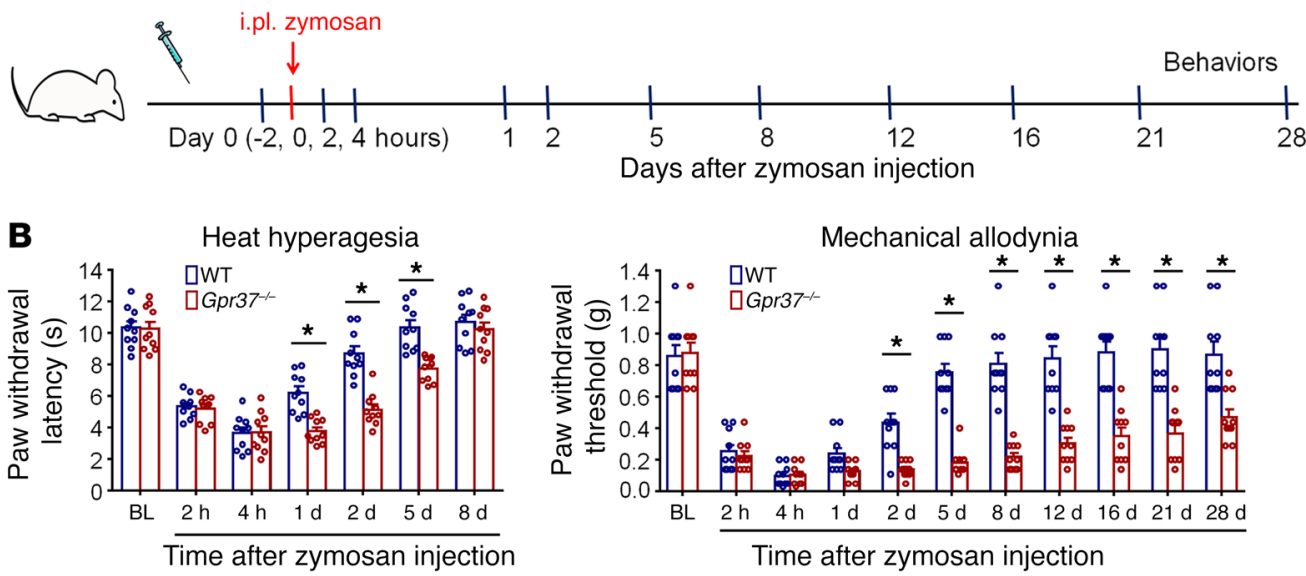

C

WT and Gpr37--- mice
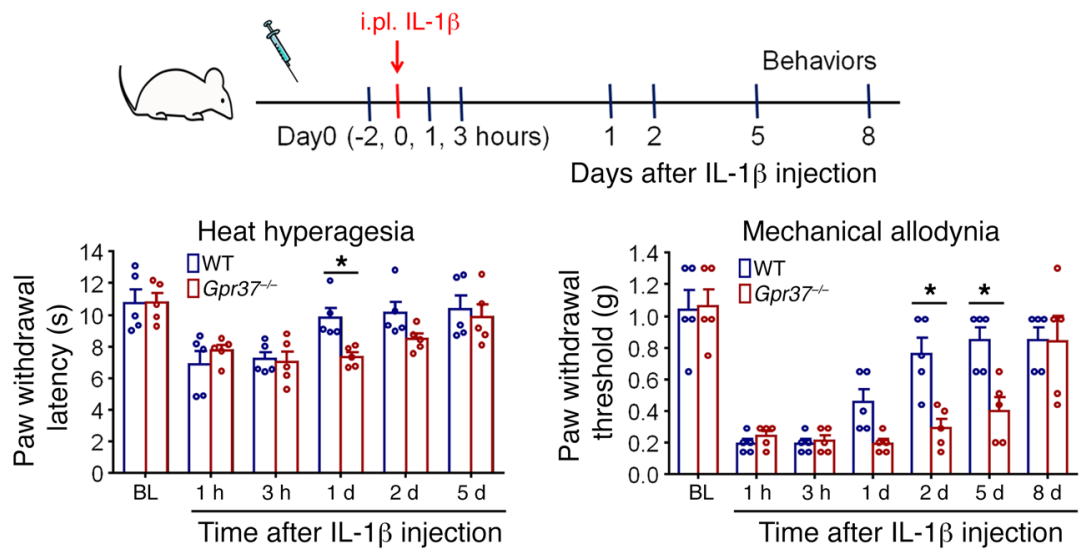

D
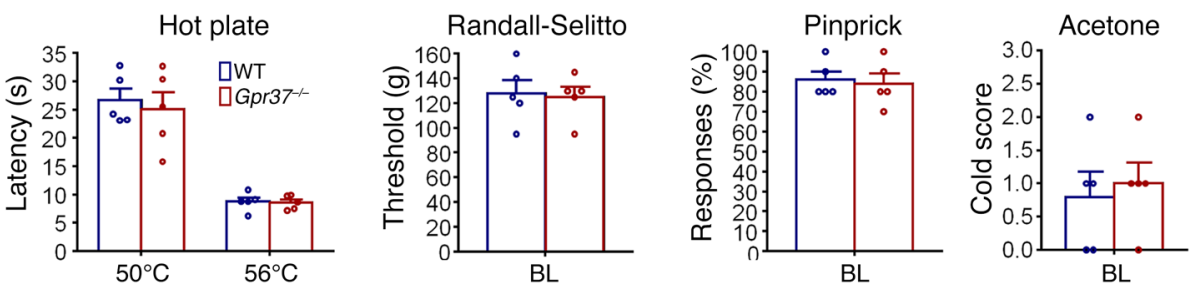

E

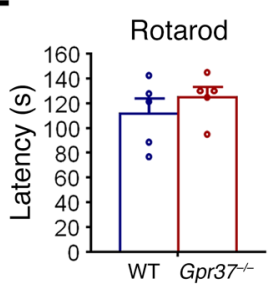

F Spontaneous pain

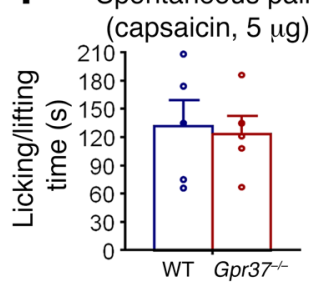

G

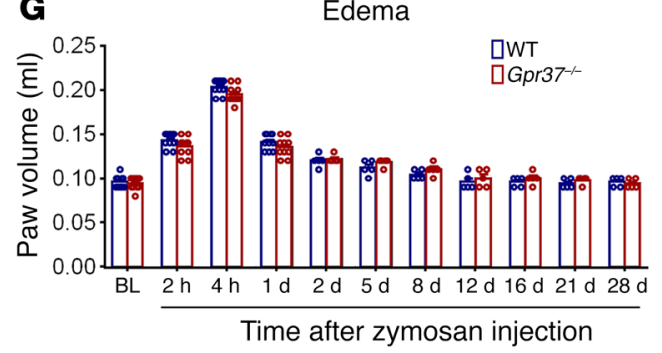

Figure 6. GPR37 is necessary for the resolution of inflammatory pain.

(A) Experimental diagram showing the timeline of i.pl. injection of zymosan and behavioral tests. (B) Zymosan-induced inflammatory pain symptoms of heat hyperalgesia and mechanical allodynia in WT and $\mathrm{Gpr} 37^{-1-}$ mice. Note that baseline (BL) pain and the onset of inflammatory pain were normal, but the resolution of inflammatory

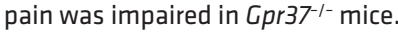
${ }^{*} P<0.05$ versus Gpr37-1-; 2 -way ANOVA. $n=10$ mice/group. (C) Heat hyperalgesia and mechanical allodynia induced by i.pl. IL-1 $\beta$ (1 ng) in WT and $\mathrm{Cpr} 7^{-1-}$ mice. Diagram shows the experimental timeline of i.pl. injection of IL-1 $\beta$ and behavioral tests. ${ }^{*} P<0.05$ versus Gpr37-1-; 2-way ANOVA. $n=5$ mice/group. (D) Baseline pain for heat sensitivity (hot plate), mechanical sensitivity (Randall-Selitto and pinprick tests), and cold sensitivity (acetone test) in WT and Gpr37-1- mice. $n=5$ mice/ group. (E) Rotarod test in WT and Gpr37-1- mice. The speed of rotation was accelerated from 4 to $40 \mathrm{rpm}$ over a 5 -minute period. $n=5$ mice/ group. (F) Acute inflammatory pain induced by capsaicin (i.pl., $5 \mu \mathrm{g}$ ) in WT and $G$ pr $37^{-1-}$ mice. $n=5$ mice/ group. (C) Zymosan-induced edema (paw swelling, revealed by paw volume) before and after zymosan injection in WT and Gpr37-1- mice. $n=5-10$ mice/group. protectins (NPD1), and maresins produce potent proresolution and antiinflammatory actions, in part by enhancing $\mathrm{M} \Phi$ phagocytic activity $(7,38)$. However, it remains unclear how SPMs regulate $\mathrm{M} \Phi$ phagocytosis, although activation of the AKT and ERK signaling pathways is important for resolvin E1-induced phagocytosis $(39,40)$. In this study, we identified GPR37 as a potential receptor for NPD1, because (a) NPD1 bound to GPR37, and (b) GPR37 mediated the NPD1-induced M $\Phi$ phagocytosis in both in vitro and in vivo assays. We found that GPR37 was expressed by MФs (including pMФs, sMФs, and DRG MФs) but not by microglia. Thus, GPR37 may serve as a new marker to distinguish microglia from МФs.

We discovered in this study that NPD1 elicited a marked $\mathrm{iCa}^{2+}$ increase in GPR37-transfected HEK293 cells and Gpr37-expressing pMФs but not in Gpr37-deficient pMФs, whereas SPMs such as RvD1 and RvE1 and their precursors DHA and EPA failed to alter 
A $\quad$ WT mice
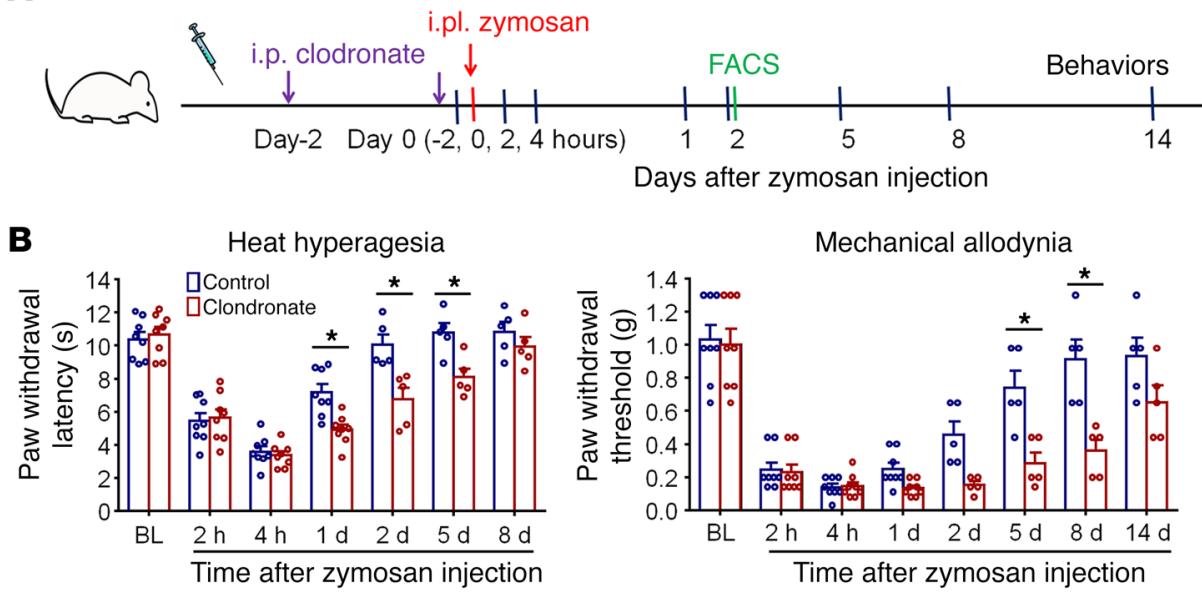

C WT and Gpr37 $7^{-1-}$ mice
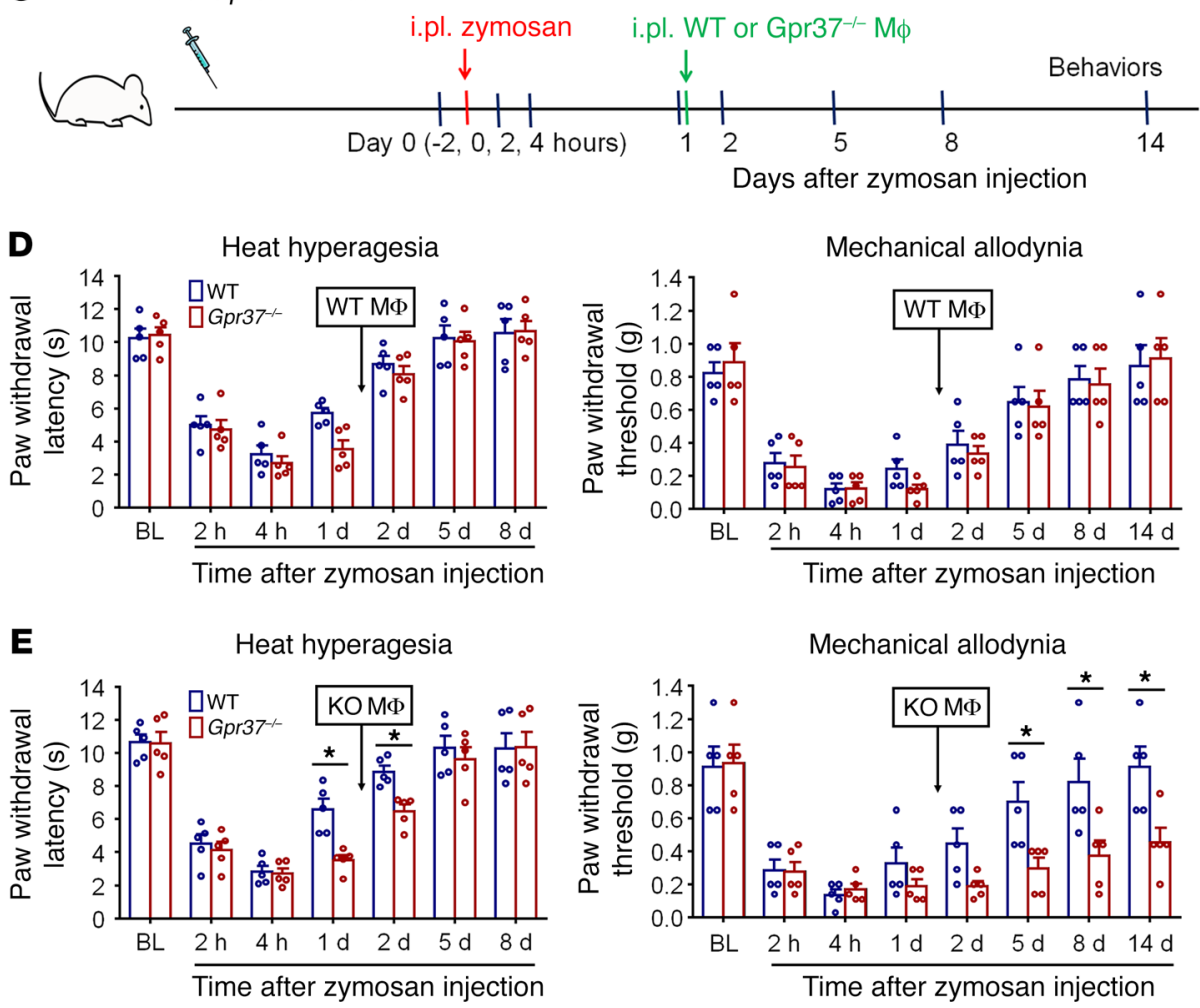

Figure 7. MФs and MФ GPR37 are critical for the resolution of inflammatory pain. (A) Experimental diagram showing the timeline of drug treatments, FACS analysis, and behavioral tests. (B) $M \Phi$ depletion with clodronate (i.p., $15 \mu \mathrm{l} / \mathrm{g}, 2$ and $48 \mathrm{~h}$ prior to the zymosan injection) delayed the resolution of heat hyperalgesia and mechanical allodynia. ${ }^{*} P<0.05$ versus control; 2 -way ANOVA. $n=$ 5-8 mice/group. (C) Experimental diagram showing the timeline of zymosan injection, adoptive transfer of MФs, and behavioral tests for $\mathbf{D}$ and $\mathbf{E}$. (D and $\mathbf{E}$ ) Adoptive transfer of WT MФs (i.pl., 50,000 cells, D) but not Gpr37-deficient МФs (КО MФs) (E) promoted the resolution of heat hyperalgesia and mechanical allodynia in $\mathrm{Gpr} 37^{-1-}$ mice. Note that the differences between $\mathrm{Cpr}_{37^{-1-}}$ and WT mice (revealed in Figure 6B) were abolished after the treatment (D). ${ }^{*} P<0.05$ versus Gpr37-I-; 2-way ANOVA. $n=5$ mice/ group. Data represent the mean $\pm \mathrm{SEM}$.
$\mathrm{iCa}^{2+}$ in $\mathrm{pM} \Phi$ s or HEK293 cells expressing respective SPM receptors (GPR32 and Chem23) (Supplemental Figure 2, A-C). GPR37 was required for the NPD1-evoked $\mathrm{iCa}^{2+}$ increase via Gi-coupled signaling. In particular, the $\mathrm{iCa}^{2+}$ increase was both sufficient and required for $\mathrm{M} \Phi$ phagocytosis. Thus, we have identified what we believe to be a unique signaling pathway through which NPD1/ GPR37 triggers phagocytosis. In contrast, a majority of the identified SPMs engage $\beta$-arrestin-2-mediated signal transduction (13). While we focused this study on pMФs, NPD1 may have additional receptors in DRG cells such as sensory neurons (10). GPR37 is known to be expressed in neurons and oligodendrocytes in the CNS (22). However, we did not find any developmental defects in primary sensory neurons or the associated neural pathways of pain in Gpr37-deficient mice. Although the Gpr37-deficient mice used in this study were global KO mice, we validated the selective role of GPR37 in МФ signaling in pМФs and sMФs. We also demonstrated a sufficient role of MФs in inflammatory pain resolution by MФ adoptive transfer experiments in vivo (Figures 7 and 8).

The zymosan model is especially suitable for investigating the resolution mechanisms of inflammatory pain. This model allows for the study of inflammation, phagocytosis, and inflammatory pain simultaneously in the inflamed hind paw skin. We have provided several lines of evidence to support a critical role of GPR37 and $M \Phi s$ in the resolution of inflammatory pain. First, a timecourse study showed that MФ numbers, zymosan phagocytosis, and GPR37 expression in the inflamed skin all peaked on day 5 during the resolution, but not the induction, phase of inflammatory pain. Second, Gpr37 deficiency and MФ depletion impaired 
A WT mice

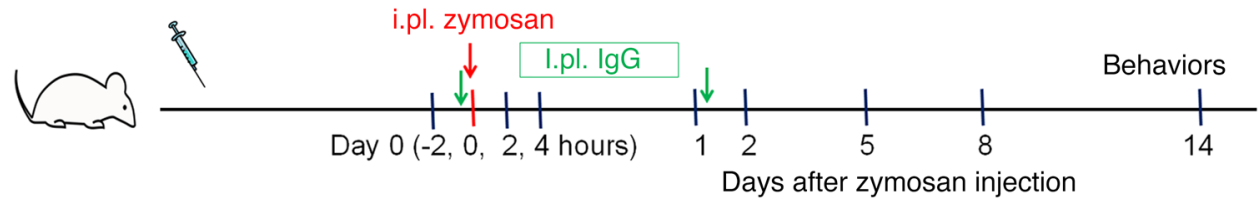

B
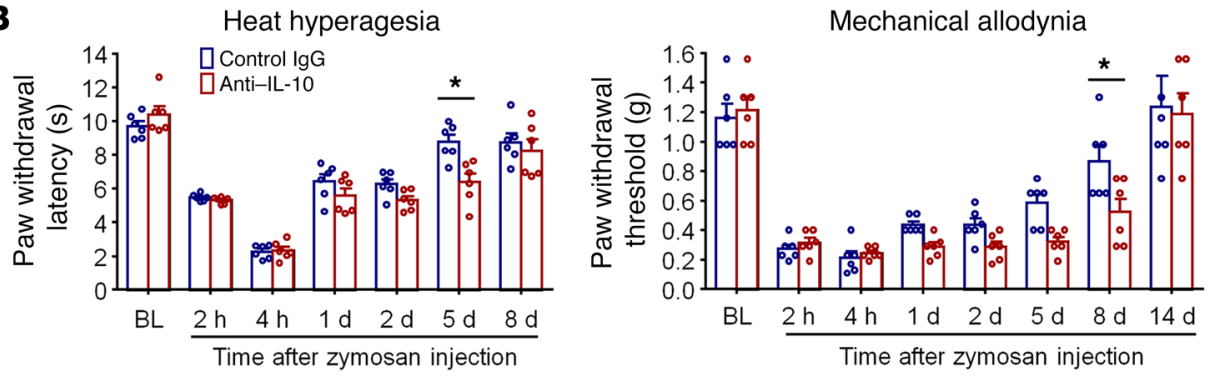

C WT and $\mathrm{Gpr} 37^{-1-}$ mice

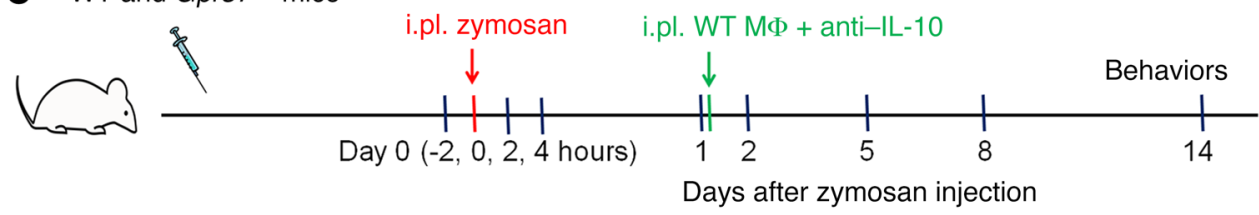

D
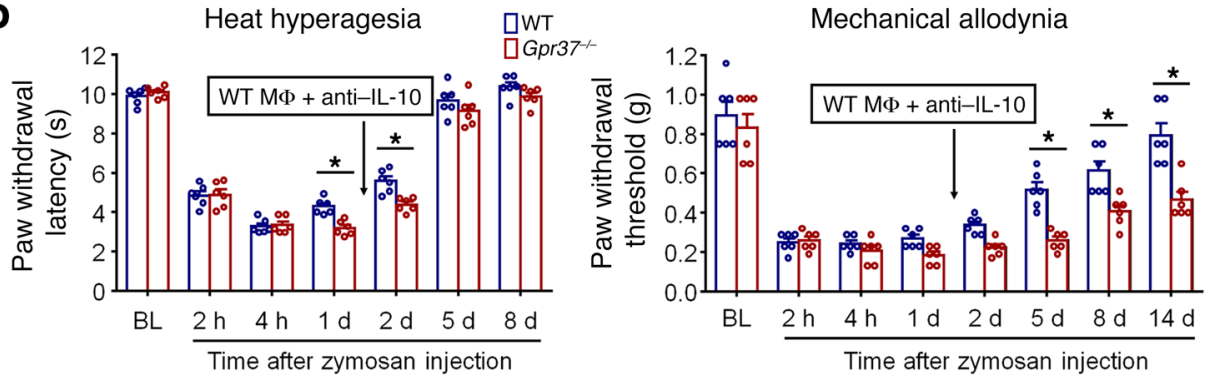

Figure 8. IL-10 contributes to the resolution of inflammatory pain by МФs.

(A) Experimental diagram showing the timeline of drug treatments and behavioral tests. (B) IL-10-neutralizing antibody (i.pl., $10 \mu \mathrm{g}$, twice, $0.5 \mathrm{~h}$ before and 1 day after the zymosan injection) delayed the resolution of heat hyperalgesia and mechanical allodynia. ${ }^{*} P<$ 0.05 versus control IgC; 2-way ANOVA. $n=6$ mice/group. (C) Experimental diagram showing the timeline of zymosan injection, adoptive transfer of MФs with IL-10-neutralizing antibody injection, and behavioral tests shown in $\mathbf{D}$. (D) IL-10-neutralizing antibody (i.pl., $10 \mu \mathrm{g}$, 1 day after zymosan injection) impaired the proresolution effects on heat hyperalgesia and mechanical allodynia induced by adoptive transfer of WT MФs (i.pl., 50,000 cells) into Gpr37-1mice. ${ }^{*} P<0.05$, WT versus Gpr37-/-; 2-way ANOVA. $n=6$ mice/group. Data represent the mean \pm SEM. the resolution of inflammatory pain by increasing the duration of pain. Third, adoptive transfer of WT, but not Gpr37-deficient, МФs was sufficient to rescue resolution deficits in Gpr37-KO mice. The accumulation of pathogens and apoptotic cell remnants often causes chronic inflammation and autoimmune disorders, which are associated with chronic pain. Therefore, GPR37-mediated $\mathrm{M} \Phi$ phagocytosis may resolve inflammatory pain by controlling the duration of inflammation. Our finding also reveals that МФs promote the resolution of inflammatory pain via production of the antiinflammatory cytokine IL-10. It remains to be determined whether $\mathrm{M} \Phi$ s resolve inflammatory pain by producing additional antiinflammatory and antinociceptive mediators.

МФs have different phenotypes, such as proinflammatory M1-like phenotypes and antiinflammatory M2 phenotypes, as well as many intermediate phenotypes that mediate the detrimental and protective actions of $\mathrm{M} \Phi s(15,16)$. Our study has shown a critical role of GPR37 is regulating MФ phenotypes. Notably, GPR37-expressing MФs exhibited proresolution M2-like phenotypes by producing lower levels of IL-1 $\beta$ but higher levels of IL-10 and TGF- $\beta$. In contrast to acute inflammation, chronic inflammation is often detrimental, leading to a host of diseases, such as chronic pain, periodontitis, atherosclerosis, rheumatoid arthritis, and even cancer (25). Thus, pharmacolog- ical enhancement of phagocytosis and phenotypic switching of МФs have translational potential in the treatment of chronic pain and inflammatory disorders. Notably, ineffective phagocytosis of amyloid- $\beta$ by MФs was found in patients with Alzheimer's disease (41), and promoting phagocytic activity could be beneficial for the prevention and treatment of the disease. NSAIDs such as COX-2 inhibitors are well known for controlling acute inflammation and inflammatory pain $(42,43)$, but they also prolong the duration of inflammation (44). The resolution of inflammation is emerging as a new therapeutic frontier (45-47). Specific targeting of GPR37 in MФs by NPD1 or its mimetics or small-molecule agonists of GPR37 may help to treat inflammatory pain and other inflammation-related disorders.

\section{Methods}

Reagents. NPD1 and RvE1 were gifts from Resolvyx Pharmaceuticals. We purchased pHrodo Red Zymosan Bioparticles Conjugate from Thermo Fisher Scientific (catalog P35364) and TX14 from Anaspec. RvD1, RvD2, DHA, EPA, and lipoxin A were purchased from Cayman Chemical. We also purchased LY294002 from Selleckchem, U0126 and IL-10-neutralizing antibody from MilliporeSigma (catalog I5145$1 \mathrm{MG}$ ), and BAPTA-AM, Fura2-AM, and Flou4-AM from Thermo Fisher Scientific. Clodronate liposomes were purchased from Liposoma B.V. 
Animals. C57BL/6 mice, Gpr37-KO mice (B6.129P2-Gpr37tm1Dgen/J, stock no. 005806), and Cx3crl-GFP mice (B6.129P-Cx3cr1 ${ }^{\mathrm{tmlLitt}} / \mathrm{J}$, stock no. 005582) were purchased from The Jackson laboratory, and the transgenic mouse lines were maintained at the Duke University animal facility. Adult 8- to 10-week-old male CD1 mice were purchased from Charles River Laboratories. Adult male mice were used for the behavioral and biochemical studies. The animals were housed under a 12-hour light/12-hour dark cycle with ad libitum access to food and water. Sample sizes were estimated on the basis of our previous studies for similar types of behavioral and biochemical analyses $(48,49)$. The numbers of mice used in different experiments are summarized in Supplemental Table 1.

Mouse models of inflammatory pain. Inflammatory pain was induced by i.pl. injection of zymosan $(1 \mu \mathrm{g} / \mu \mathrm{l}, 20 \mu \mathrm{l})$, IL-1 $\beta$ ( $1 \mathrm{ng}, 20$ $\mu \mathrm{l})$, or capsaicin $(5 \mu \mathrm{g}, 10 \mu \mathrm{l})$ into a hind paw.

Drug injection. For local i.pl. injection, drugs $(20 \mu \mathrm{l})$ were injected using a Hamilton microsyringe with a 30-gauge needle.

ISH. We used probes directed against mouse Gpr37 designed by Advanced Cell Diagnostics (RNAscope Probe-Mm-Gpr37, catalog 319291) and performed an RNAscope Fluorescent Multiplex Fluorescent assay according to the manufacturer's instructions. Skin sections (20- $\mu \mathrm{m}$ thick) were used for ISH. Prehybridization, hybridization, and washing were performed according to the manufacturer's instructions.

LacZ staining. Mice were anesthetized and perfused with PBS followed by $4 \%$ paraformaldehyde (PFA). Spinal cord, skin, and brain tissues were collected and post-fixed with $1 \%$ glutaraldehyde and $2 \%$ PFA for 2 hours at $4^{\circ} \mathrm{C}$ and then transferred to $30 \%$ sucrose overnight at $4^{\circ} \mathrm{C}$. Tissues were washed with PBS containing $2 \mathrm{mM} \mathrm{MgCl}_{2}$, embedded in OCT, and sectioned (20- to $30-\mu \mathrm{m}$ thick) in a cryostat. The sections were washed with $2 \mathrm{mM} \mathrm{MgCl}_{2}$ and immediately used for LacZ staining. The staining was processed for 1 to 16 hours at $37^{\circ} \mathrm{C}$ in a staining solution containing $5 \mathrm{mM}$ potassium ferrocyanide, $5 \mathrm{mM}$ potassium ferricyanide, $0.01 \%$ sodium deoxycholate, $0.02 \%$ NP40, $2 \mathrm{mM} \mathrm{MgCl}$, and $1 \mathrm{mg} / \mathrm{ml} \mathrm{X}$-gal.

IHC and quantification. After appropriate survival times, the mice were deeply anesthetized with isoflurane and perfused through the ascending aorta with PBS, followed by $4 \%$ PFA. After the perfusion, the L4-L5 DRGs and hind paw glabrous skins were removed and postfixed in the same fixative overnight. DRG and skin tissue sections $(10$ or $20 \mu \mathrm{m})$ and free-floating spinal cord and skin sections (30- $\mu \mathrm{m}$-thick) were cut in a cryostat. The sections were blocked with $2 \%$ goat or donkey serum for 1 hour at room temperature and then incubated overnight at $4^{\circ} \mathrm{C}$ with the following primary antibodies: antiGPR37 (mouse, 1:500, Santa Cruz Biotechnology, catalog sc390110), anti-CD68 (rat, 1:500, BioLegend, catalog 137001), anti-F4/80 (rat, 1:1000, eBioscience, catalog 14-4801-82), anti-IBA1 (rabbit, 1:1,000, Wako, catalog 019-19741), anti-Gr-1 (rat, 1:1,000, BioLegend, catalog 127601), anti-CGRP (goat, 1:1,000; Bio-Rad, catalog AB_2290729), anti-NF200 (mouse, 1:1,000, MilliporeSigma, catalog AB_477257), and anti-PGP9.5 (rabbit, 1:2,000, UltraClone, catalog AB_2313685) antibodies. After washing in PBS, the sections were then incubated with cyanine 3- (Сy3-), cyanine 5- (Cy5-), or FITC-conjugated secondary antibodies (1:400, Jackson ImmunoResearch) for 2 hours at room temperature. For double immunofluorescence, sections were incubated with a mixture of polyclonal and monoclonal primary antibodies, followed by a mixture of Cy3- and FITC-conjugated secondary antibodies or FITC-conjugated IB4 $(10 \mu \mathrm{g} / \mathrm{ml}$; MilliporeSigma). In some cases, DAPI (1:1,000, Vector Laboratories, catalog H-1200) was used to stain cell nuclei in the tissue sections. The stained sections were examined with a Nikon fluorescence microscope, and images were captured with a CCD SPOT camera (SPOT Imaging). For high-resolution images, sections were also examined under a Zeiss 510 inverted confocal microscope. To confirm the specificity of the GPR37 antibody, immunostaining was also conducted in Gpr37-KO mice. To quantify immunofluorescence intensity in skin sections from WT and KO mice, 3-5 sections were included per animal, and the fluorescence intensity was measured using Image J software (NIH).

ELISA. Mouse ELISA kits for IL-1 $\beta$, TGF- $\beta$, and IL-10 were purchased from R\&D Systems (catalog MLB00C for IL-1 $\beta$, catalog MB100B for TGF- $\beta$, and catalog M1000B for IL-10). ELISA was performed using culture media and hind paw skin tissues. Cultured cells and tissues were homogenized in a lysis buffer containing protease and phosphatase inhibitors. For each ELISA assay, $50 \mu \mathrm{g}$ protein or $50 \mu \mathrm{l}$ culture medium was used. ELISA was conducted according to the manufacturer's instructions. The standard curve was included in each experiment.

Quantitative real-time RT-PCR. Hind paw skins of mice were collected at different times points of zymosan injection. Total RNA from the skins and cultures was extracted using the Direct-zol RNA MiniPrep Kit (Zymo Research), and 0.5-1 $\mu \mathrm{g}$ RNA was reverse transcribed using the iScript cDNA Synthesis Kit (Bio-Rad). Specific primers including the GAPDH control were designed using IDT SciTools Real-Time PCR software. We performed gene-specific mRNA analyses using the MiniOpticon Real-Time PCR System (Bio-Rad). Quantitative PCR amplification reactions contained the same amount of reverse transcription product, including $7.5 \mu$ of $2 \times$ iQSYBR Green Mix (Bio-Rad) and 100-300 nM forward and reverse primers in a final volume of $15 \mu$ l. The primer sequences are listed in Supplemental Table 2. Primer efficiency was obtained from the standard curve and integrated for the calculation of relative gene expression, which was based on real-time PCR threshold values of different transcripts.

Western blot analysis. Protein samples were prepared in the same way as for ELISA analysis, and $30 \mu$ g protein was loaded for each lane and separated by $10 \%$ SDS-PAGE gel. After the transfer, the blots were incubated overnight at $4^{\circ} \mathrm{C}$ with polyclonal or monoclonal antibodies against GPR37 (1:2,000, mouse, Santa Cruz Biotechnology, cata$\log$ sc-390110). For the loading control, the blots were probed with GAPDH antibody (1:5,000, rabbit, BioWorld, catalog AP0063). These blots were further incubated with an HRP-conjugated secondary antibody and developed in ECL solution (Pierce, Thermo Fisher Scientific). Chemiluminescence signal was revealed by Bio-Rad ChemiDoc XRS for 1 to 10 minutes. Specific bands were evaluated according to apparent molecular sizes.

HEK cell culture and transfection. The HEK293 flip-in cell line was purchased from the Duke Cell Culture Facility. Cells were cultured in high glucose (4.5 g/l) DMEM containing 10\% (v/v) FBS (Gibco, Thermo Fisher Scientific). Transfection ( $2 \mu \mathrm{g}$ cDNA) was performed with Lonza electroporation at $70 \%$ confluency, and the transfected cells were cultured in the same growth medium for 48 hours before use. The hGPR37-V5 pLenti304 plasmids were obtained from the DNASU Plasmid Repository, and hGPR32, hChemR23, and hALX pcDNA3 plasmids were provided by Charles Serhan (Harvard Medical School, Boston, Massachusetts, USA).

Dot blot assay for lipid-protein binding. Lipid membrane coating and protein overlay assays were conducted as previously described (50). Ethanol and chloroform-soluble fatty acids were directly loaded 
onto a hydrophobic PVDF membrane (MilliporeSigma). The membrane was coated with NPD1, TX14, and RvE1. The fatty acid-coated membranes were dried and blocked with 1\% BSA. The HEK293 cells were transfected with GPR37 cDNA with a V5 tag (GPR37-V5) or with an empty vector (mock transfection) using Lipofectamine 2000 (Invitrogen, Thermo Fisher Scientific). Cell lysates were incubated overnight with the coating membrane at $4^{\circ} \mathrm{C}$, and the binding was detected by anti-V5-tagged antibody (mouse, 1:1,000, Thermo Fisher Scientific, catalog 46-0705). The blots were further incubated with an HRP-conjugated secondary antibody and developed in ECL solution (Pierce, Thermo Fisher Scientific). The intensity of lipid-protein binding was evaluated using Image J software.

$p M \Phi$ culture. $\mathrm{pM} \Phi$ s were collected by peritoneal lavage with $10 \mathrm{ml}$ warm PBS containing $1 \mathrm{mM}$ EDTA. Cells were incubated in DMEM supplemented with $10 \% \mathrm{FBS}$ at $37^{\circ} \mathrm{C}$ for 2 hours in a Petri dish and washed with PBS to eliminate nonadherent cells. The adherent cells were used as

Calcium imaging. HEK293 cells, with or without transfection of human GPR37, GPR32, Chem23, or ALX1 cDNAs, were loaded with 5 $\mu \mathrm{M}$ Fura-2AM (Invitrogen, Thermo Fisher Scientific) for 1 hour and then resuspended in normal external buffer $(140 \mathrm{mM} \mathrm{NaCl}, 5 \mathrm{mM}$ $\mathrm{KCl}, 2 \mathrm{mM} \mathrm{CaCl}$, $2 \mathrm{mM} \mathrm{MgCl}$, $10 \mathrm{mM}$ HEPES, titrated to $\mathrm{pH} 7.4$ with $\mathrm{NaOH}$ ) or external $\mathrm{Ca}^{2+}$-free buffer (140 mM NaCl, $2 \mathrm{mM} \mathrm{MgCl} 2,5 \mathrm{mM}$ EGTA, 10 mM HEPES, titrated to $\mathrm{pH} 7.4$ with $\mathrm{NaOH}$ ). pMФs, collected from WT and Gpr37-KO mice, were loaded with $5 \mu \mathrm{M}$ Fluo-4AM (Invitrogen, Thermo Fisher Scientific) for 1 hour and then resuspended in normal external buffer. Images of the HEK293 cells with an excitation wavelength of $340 \mathrm{~nm}$ and $380 \mathrm{~nm}$ or МФs with an excitation wavelength of $488 \mathrm{~nm}$ were captured with a cooled Digital CMOS camera (ORCA-Flash 4.0, Hamamatsu Photonics). The ratio of fluorescence intensity of the 2 wavelengths in each experiment was analyzed using MetaFluor software(Molecular Devices). The Shutter speed and wavelength were controlled by the Lambda DG-4 system (Sutter Instrument Company). Values from each experiment were normalized to the baseline ratio of 340:380 $\mathrm{nm}$ or the basal intensity for MФs. Quantification of calcium levels was conducted on 72 to 250 HEK293 cells or MФs/ condition/well, and at least 3 cultures were included.

Isolation of skin cells. Skin tissues from noninflamed and inflamed hind paws were cut into small strips of 1 to $2 \mathrm{~mm}$ thickness and placed in $1 \mathrm{mg} / \mathrm{ml}$ collagenase A and $2.4 \mathrm{U} / \mathrm{ml}$ Dispase II (Roche Applied Sciences) in HBSS. Tissues were incubated at $37^{\circ} \mathrm{C}$ with continuous shaking at $200 \mathrm{rpm}$ for 90 minutes. The skin cells were triturated by pipette, washed with HBSS and 0.5\% BSA, and filtered through a $70-\mu \mathrm{m}$ mesh and then treated with $1 \times \mathrm{RBC}$ lysis buffer (BioLegend).

Flow cytometry. Surface expression of GPR37, F4/80, CD11b, and Ly-6G was determined by flow cytometry, as we previously described (51). The dissociated skin or peritoneal cells were counted, and $2 \times$ $10^{6}$ cells per sample were stained with a Zombie Violet Fixable Viability Kit (BioLegend, catalog 423114) according to the manufacturer's instructions. All dissociated cells were blocked with Fc receptor staining buffer (1\% anti-mouse CD16/CD32, 2.4 G2, 2\% FBS, 5\% NRS, and $2 \%$ NMS in HBSS; BD Biosciences) and then stained with a standard panel of antibodies. After staining, cells were washed in PBS with EDTA. The flow cytometric events were acquired in a BD FACSCanto II flow cytometer using BD FACSDiva 8 software (BD Biosciences). Data were analyzed using Cytobank software (https://www.cytobank. org/cytobank). For neutrophil phagocytosis by MФs in skin, dissociat- ed cells were labeled with FITC-conjugated anti-mouse F4/80 antibody, permeabilized with $0.1 \%$ Triton X-100, and then labeled with APC-Cy7-anti-Ly6G antibody and analyzed by flow cytometry. See Supplemental Table 3 for details on the antibodies used.

Phagocytosis assay. The phagocytosis assay was modified from a previously described protocol (27). pHrodo Red Zymosan Bioparticles (3- $\mu \mathrm{m}$ diameter, Thermo Fisher Scientific, catalog P35364) were rinsed and reconstituted in RPMI medium. Particles were centrifuged ( 1 min at $100 \mathrm{~g}$ ) onto adherent $\mathrm{pM \Phi s} \mathrm{at} \mathrm{a} \mathrm{ratio} \mathrm{of} \mathrm{10:1} \mathrm{to} \mathrm{synchronize}$ binding and internalization. After a 30 -minute incubation at $37^{\circ} \mathrm{C}$, nonadherent beads were removed with cold PBS, and cells were fixed with $2 \%$ formalin. Four optic fields were photographed by epifluorescence microscopy. The quantification of zymosan particles ingested by MФs was conducted on 38 to 344 MФs per condition or well, and triplicates were included for statistical analysis.

Behavioral tests in mice. Several behavioral assessments were conducted in a blinded manner. For spontaneous pain, we measured the time (seconds) mice spent licking or flinching the affected hind paws over a 5-minute period after the capsaicin injection (i.pl. $5 \mu \mathrm{g}$ ). To evaluate mechanical pain using the von Frey test, animals were habituated to the testing environment daily for at least 2 days before baseline assessment. The room temperature and humidity remained stable for all experiments. To test mechanical sensitivity, we confined mice in boxes placed on an elevated metal mesh floor and stimulated their hind paws with a series of von Frey hairs with logarithmically increasing stiffness (0.02-2.56 g, Stoelting), presented perpendicularly to the central plantar surface. We determined the $50 \%$ paw withdrawal threshold by Dixon's up-down method (48). We used a Randall-Selitto analgesy meter (Ugo Basile) to examine mechanical sensitivity by applying ascending pressure to the tail of a mouse and determined the mechanical pain threshold when the animal showed a clear sign of discomfort or desire to escape, with a cutoff threshold of $250 \mathrm{~g}$ to avoid tissue damage (52). Thermal sensitivity was tested using a Hargreaves radiant heat apparatus (IITC Life Science) (53). The basal paw withdrawal latency was adjusted to 9 to 12 seconds, with a cutoff of 20 seconds to prevent tissue damage. Thermal sensitivity was also tested using a hot plate (Bioseb in Vivo Research Instrument). The surface temperature of the plate was controlled over a range of $50^{\circ} \mathrm{C}$ to $56^{\circ} \mathrm{C}$. To test cold sensitivity, a drop of acetone was applied to the ventral surface of a hind paw, and the mouse's response was observed for 30 seconds after acetone application (54). Responses to acetone were graded according to the following 4-point scale: 0 , no response; 1 , quick withdrawal or flick of the paw; 2, prolonged withdrawal or flicking; 3, repeated flicking with licking. A Rotarod system (IITC Life Science) was used to assess motor function (48). Mice were tested in 3 trials separated by 10-minute intervals. During the tests, the speed of rotation was accelerated from 4 to $40 \mathrm{rpm}$ over a 5 -minute period. The falling latency was recorded. Paw swelling (edema) after zymosan injection was determined by water displacement plethysmometer (Ugo Basile) (55). The plethysmometer is a microcontrolled volume meter specially designed for the accurate measurement of mouse paw swelling.

Statistics. All data in the figures are expressed as the mean \pm SEM. The sample size for each experiment was based on our previous studies involving such experiments $(48,49)$. Statistical analyses were performed using GraphPad Prism 6.0 (GraphPad Software). Biochemical and behavioral data were analyzed using a 2-tailed Student's $t$ test (2-group comparisons) or 2-way ANOVA followed by 
Bonferroni's post hoc test. The criterion for statistical significance was a $P$ value of less than 0.05 .

Study approval. The present studies in animals were reviewed and approved by the IACUCs of Duke University and Zhejiang University. All animal procedures were conducted in accordance with the NIH's Guide for the Care and Use of Laboratory Animals (National Academies Press, 2011).

\section{Author contributions}

SB conducted calcium imaging, phagocytosis, flow cytometry, and some binding assays. YKX contributed to binding and Western blot analyses and behavioral and histochemical experiments under the supervision of ZZX. ZJZ performed histochemical experiments. ZW helped with calcium imaging. ZZX performed behavioral tests and some histochemical studies. ZZX and SB analyzed the data and organized the figures. ZZX and RRJ designed the study. RRJ, ZZX, and SB wrote the manuscript.

\section{Acknowledgments}

We thank Charles Serhan for providing CMKLR1 (ChemR23) GPR32, and ALX1 cDNAs. This study was supported by NIH R01 grants NS87988, NS89479, DE17794, and DE22743 (to RRJ) and NIH R21 grant NS91779 (to ZZX and RRJ); the National Natural Science Foundation of China (NSFC) (31771162, to ZZX); and the Zhejiang Provincial Natural Science Foundation of China (LZ18C090002, to ZZX). ZZX was also supported by the K.C. Wong Education Foundation.

Address correspondence to: Zhen-Zhong $\mathrm{Xu}$, Department of Physiology, Center of Neuroscience, Zhejiang University School of Medicine, Hangzhou, China. Email: xuzz@zju.edu.cn. Or to: Ru-Rong Ji, PhD, Department of Anesthesiology, Duke University Medical Center, Durham, North Carolina, 27710, USA. Phone: 919.684.9387; Email: ru-rong.ji@duke.edu.
1. Chiu IM, von Hehn CA, Woolf CJ. Neurogenic inflammation and the peripheral nervous system in host defense and immunopathology. Nat Neurosci. 2012;15(8):1063-1067.

2. Ji RR, Chamessian A, Zhang YQ. Pain regulation by non-neuronal cells and inflammation. Science. 2016;354(6312):572-577.

3. Chiu IM, et al. Bacteria activate sensory neurons that modulate pain and inflammation. Nature. 2013;501(7465):52-57.

4. Ghasemlou N, Chiu IM, Julien JP, Woolf CJ. $\mathrm{CD} 11 \mathrm{~b}^{+} \mathrm{Ly}_{6 \mathrm{G}^{-}}$myeloid cells mediate mechanical inflammatory pain hypersensitivity. Proc Natl Acad Sci U S A. 2015;112(49):E6808-E6817.

5. Sommer C, Kress M. Recent findings on how proinflammatory cytokines cause pain: peripheral mechanisms in inflammatory and neuropathic hyperalgesia. Neurosci Lett. 2004;361(1-3):184-187.

6. Binshtok AM, et al. Nociceptors are interleukin-1 $\beta$ sensors. J Neurosci. 2008;28(52):14062-14073.

7. Serhan CN, Chiang N, Van Dyke TE. Resolving inflammation: dual anti-inflammatory and pro-resolution lipid mediators. Nat Rev Immunol. 2008;8(5):349-361.

8. Xu ZZ, et al. Resolvins RvE1 and RvD1 attenuate inflammatory pain via central and peripheral actions. Nat Med. 2010;16(5):592-597.

9. Sommer C, Birklein F. Fighting off pain with resolvins. Nat Med. 2010;16(5):518-520.

10. Park CK, Lü N, Xu ZZ, Liu T, Serhan CN, Ji RR. Resolving TRPV1- and TNF- $\alpha$-mediated spinal cord synaptic plasticity and inflammatory pain with neuroprotectin D1. J Neurosci. 2011;31(42):15072-15085.

11. Xu ZZ, et al. Neuroprotectin/protectin D1 protects against neuropathic pain in mice after nerve trauma. Ann Neurol. 2013;74(3):490-495.

12. Arita M, Ohira T, Sun YP, Elangovan S, Chiang $\mathrm{N}$, Serhan CN. Resolvin E1 selectively interacts with leukotriene B4 receptor BLT1 and ChemR23 to regulate inflammation. JImmunol. 2007;178(6):3912-3917.

13. Krishnamoorthy S, et al. Resolvin D1 binds human phagocytes with evidence for proresolving receptors. Proc Natl Acad Sci U S A. 2010;107(4):1660-1665.

14. Serhan CN. Resolution phase of inflammation: novel endogenous anti-inflammatory and proresolving lipid mediators and pathways. Annu Rev Immunol. 2007;25:101-137.

15. Aderem A, Underhill DM. Mechanisms of phagocytosis in macrophages. Annu Rev Immunol. 1999;17:593-623.

16. Mosser DM, Edwards JP. Exploring the full spectrum of macrophage activation. Nat Rev Immunol.2008;8(12):958-969.

17. Marazziti D, Golini E, Gallo A, Lombardi MS, Matteoni R, Tocchini-Valentini GP. Cloning of GPR37, a gene located on chromosome 7 encoding a putative G-protein-coupled peptide receptor, from a human frontal brain EST library. Genomics. 1997;45(1):68-77.

18. Leng N, Gu G, Simerly RB, Spindel ER. Molecular cloning and characterization of two putative $G$ protein-coupled receptors which are highly expressed in the central nervous system. Brain Res Mol Brain Res. 1999;69(1):73-83.

19. Murakami T, et al. Pael-R is accumulated in Lewy bodies of Parkinson's disease. Ann Neurol. 2004;55(3):439-442.

20. Marazziti D, Mandillo S, Di Pietro C, Golini E, Matteoni R, Tocchini-Valentini GP. GPR37 associates with the dopamine transporter to modulate dopamine uptake and behavioral responses to dopaminergic drugs. Proc Natl Acad Sci U S A. 2007;104(23):9846-9851.

21. Fujita-Jimbo E, et al. Mutation in Parkinson disease-associated, G-protein-coupled receptor 37 (GPR37/PaelR) is related to autism spectrum disorder. PLoS ONE. 2012;7(12):e51155.

22. Yang HJ, Vainshtein A, Maik-Rachline G, Peles E. $G$ protein-coupled receptor 37 is a negative regulator of oligodendrocyte differentiation and myelination. Nat Commun. 2016;7:10884.

23. Jolivalt CG, Ramos KM, Herbetsson K, Esch FS, Calcutt NA. Therapeutic efficacy of prosaposin-derived peptide on different models of allodynia. Pain. 2006;121(1-2):14-21.

24. Meyer RC, Giddens MM, Schaefer SA, Hall RA. GPR37 and GPR37L1 are receptors for the neuroprotective and glioprotective factors prosaptide and prosaposin. Proc Natl Acad Sci US A. 2013;110(23):9529-9534.

25. Ji RR, Xu ZZ, Gao YJ. Emerging targets in neu- roinflammation-driven chronic pain. Nat Rev Drug Discov. 2014;13(7):533-548.

26. Meyer RC, Giddens MM, Coleman BM, Hall RA. The protective role of prosaposin and its receptors in the nervous system. Brain Res. 2014;1585:1-12.

27. Link TM, Park U, Vonakis BM, Raben DM, Soloski MJ, Caterina MJ. TRPV2 has a pivotal role in macrophage particle binding and phagocytosis. Nat Immunol. 2010;11(3):232-239.

28. Beceiro S, et al. TRPM2 ion channels regulate macrophage polarization and gastric inflammation during Helicobacter pylori infection. $\mathrm{Muco}$ sal Immunol. 2017;10(2):493-507.

29. Zou J, et al. A differential role of macrophage TRPM2 channels in $\mathrm{Ca}^{2+}$ signaling and cell death in early responses to $\mathrm{H}_{2} \mathrm{O}_{2}$. Am J Physiol, Cell Physiol. 2013;305(1):C61-C69.

30. Huang NN, et al. Canonical and noncanonical g-protein signaling helps coordinate actin dynamics to promote macrophage phagocytosis of zymosan. Mol Cell Biol. 2014;34(22):4186-4199.

31. Gantner BN, Simmons RM, Canavera SJ, Akira S, Underhill DM. Collaborative induction of inflammatory responses by dectin-1 and Toll-like receptor 2. JExp Med. 2003;197(9):1107-1117.

32. Meller ST, Gebhart GF. Intraplantar zymosan as a reliable, quantifiable model of thermal and mechanical hyperalgesia in the rat. Eur J Pain. 1997;1(1):43-52.

33. Schwab JM, Chiang N, Arita M, Serhan CN. Resolvin E1 and protectin D1 activate inflammation-resolution programmes. Nature. 2007;447(7146):869-874.

34. Murray PJ, Wynn TA. Protective and pathogenic functions of macrophage subsets. Nat Rev Immunol. 2011;11(11):723-737.

35. Van Rooijen N, Sanders A. Liposome mediated depletion of macrophages: mechanism of action, preparation of liposomes and applications. J Immunol Methods. 1994;174(1-2):83-93.

36. McKelvey R, Berta T, Old E, Ji RR, Fitzgerald M. Neuropathic pain is constitutively suppressed in early life by anti-inflammatory neuroimmune regulation. J Neurosci. 2015;35(2):457-466.

37. Krukowski K, et al. CD8+ T cells and endogenous IL-10 are required for resolution of chemo- 
therapy-induced neuropathic pain. J Neurosci. 2016;36(43):11074-11083.

38. Chiang N, et al. Infection regulates pro-resolving mediators that lower antibiotic requirements. Nature. 2012;484(7395):524-528.

39. El Kebir D, Gjorstrup P, Filep JG. Resolvin E1 promotes phagocytosis-induced neutrophil apoptosis and accelerates resolution of pulmonary inflammation. Proc Natl Acad Sci U S A. 2012;109(37):14983-14988.

40. Ohira T, Arita M, Omori K, Recchiuti A, Van Dyke $\mathrm{TE}$, Serhan CN. Resolvin E1 receptor activation signals phosphorylation and phagocytosis. J Biol Chem. 2010;285(5):3451-3461.

41. Fiala M, et al. Ineffective phagocytosis of amyloid-beta by macrophages of Alzheimer's disease patients. JAlzheimers Dis. 2005;7(3):221-232.

42. Malmberg AB, Yaksh TL. Hyperalgesia mediated by spinal glutamate or substance $P$ receptor blocked by spinal cyclooxygenase inhibition. Science. 1992;257(5074):1276-1279.

43. Samad TA, et al. Interleukin-1beta-mediated induction of Cox-2 in the CNS contributes to inflammatory pain hypersensitivity. Nature. 2001;410(6827):471-475.

44. Gilroy DW, Colville-Nash PR, Willis D, Chivers J, Paul-Clark MJ, Willoughby DA. Inducible cyclooxygenase may have anti-inflammatory properties. Nat Med.1999;5(6):698-701.

45. Fullerton JN, Gilroy DW. Resolution of inflammation: a new therapeutic frontier. Nat Rev Drug Discov. 2016;15(8):551-567.

46. Perretti M, Cooper D, Dalli J, Norling LV. Immune resolution mechanisms in inflammatory arthritis. Nat Rev Rheumatol. 2017;13(2):87-99.

47. Perretti M, Leroy X, Bland EJ, Montero-Melendez T. Resolution pharmacology: opportunities for therapeutic innovation in inflammation. Trends Pharmacol Sci. 2015;36(11):737-755.

48. Chen G, Park CK, Xie RG, Ji RR. Intrathecal bone marrow stromal cells inhibit neuropathic pain via TGF- $\beta$ secretion. J Clin Invest. 2015;125(8):3226-3240.

49. Berta T, et al. Extracellular caspase- 6 drives murine inflammatory pain via microglial TNF- $\alpha$ secretion. JClin Invest. 2014;124(3):1173-1186.

50. Dowler S, Kular G, Alessi DR. Protein lipid overlay assay. Sci STKE. 2002;2002(129):pl6.

51. Liu XJ, et al. Nociceptive neurons regulate innate and adaptive immunity and neuropathic pain through MyD88 adapter. Cell Res. 2014;24(11):1374-1377.

52. Liu T, et al. TLR3 deficiency impairs spinal cord synaptic transmission, central sensitization, and pruritus in mice. J Clin Invest. 2012;122(6):2195-2207.

53. Hargreaves K, Dubner R, Brown F, Flores C, Joris $\mathrm{J}$. A new and sensitive method for measuring thermal nociception in cutaneous hyperalgesia. Pain. 1988;32(1):77-88.

54. Chen $\mathrm{G}$, et al. $\beta$-arrestin-2 regulates NMDA receptor function in spinal lamina II neurons and duration of persistent pain. Nat Commun. 2016;7:12531.

55. Chen G, et al. PD-L1 inhibits acute and chronic pain by suppressing nociceptive neuron activity via PD-1. Nat Neurosci. 2017;20(7):917-926. 